\title{
Lazaroa
}

\section{Syntaxonomical update on the relict groves of Scots pine (Pinus sylvestris var. iberica) and Spanish black pine (Pinus nigra subsp. salzmannii) in the Gredos range (central Spain)}

\author{
José Antonio López-Sáez ${ }^{1}$, Daniel Sánchez-Mata² \& Rosario G. Gavilán²
}

Received: 30 September 2016 / Accepted: 4 November 2016

\begin{abstract}
Pure and mixed high-mountain natural pine forest vegetation of the Gredos range (central Spain) was studied using 57 relevés and multivariate analyses (hierarchical cluster analysis and detrended correspondence analysis). Classification of the relevés resulted in 7 vegetation units, 3 of which were ranked as associations and only one as subassociation. DCA diagrams of relevés and taxa indicated that floristic differentiation was attributed mainly to factors such as altitude. Differential taxa of vegetation units were chosen based on their phi coefficient values. The statistic discrimination of some of these vegetation units has allowed us to propose a new association (Genisto cinerascentisPinetum salzmannii) and a new subassociation of other community described in a previous paper (Echinosparto barnadesii-Pinetum ibericae pinetosum salzmannii).

Keywords: Pinus nigra subsp. salzmannii; Pinus sylvestris var. iberica; Gredos range; Phytosociology; Multivariate Analyses; Community Ecology.
\end{abstract}

[es] Estudio sintaxonómico de los bosques relícticos de pino silvestre (Pinus sylvestris var. iberica) y pino negro (Pinus nigra subsp. salzmannii) en la sierra de Gredos (centro de España)

Resumen. Se han estudiado pinares naturales puros y mixtos en la alta montaña de la Sierra de Gredos (centro de España) mediante 57 inventarios florísticos y análisis multivariantes (análisis de cluster jerárquico y análisis de correspondencias sin tendencia). La clasificación de los inventarios deparó 7 unidades de vegetación, 3 de las cuales fueron clasificadas a nivel de asociación y otra como subasociación. Los diagramas del DCA de inventarios y taxones indican que la diferenciación florística puede atribuirse mayoritariamente a factores tales como la altitud. Los taxones diagnósticos de cada unidad de vegetación fueron elegidos de acuerdo a los valores de su coeficiente phi. La discriminación estadística de las unidades de vegetación nos hace proponer una nueva asociación (Genisto cinerascentisPinetum salzmannii) y una nueva subasociación de una comunidad descrita en un trabajo anterior (Echinosparto barnadesii-Pinetum ibericae pinetosum salzmannii).

Palabras clave: Pinus nigra subsp. salzmannii; Pinus sylvestris var. iberica; sierra de Gredos; Fitosociología; análisis multivariantes; ecología de las comunidades vegetales.

\section{Introduction}

Pine species are important components of many natural ecosystems across the Mediterranean, especially in mountain regions (Barbero \& al., 1998), and their historical and genetic dynamics may therefore have commu- nity-level consequences (Soto \& al., 2010). In the Iberian Peninsula, Pinus nigra subsp. salzmannii and $P$. sylvestris are among the commonest species in mountainous areas, both in the Atlantic and the Mediterranean biogeographical regions (Blanco Castro \& al., 1997). The interest in the Gredos mountain

\footnotetext{
Grupo de Investigación Arqueobiología, Instituto de Historia, CCHS, CSIC. Albasanz 26-28. E-28037 Madrid. Spain. E-mail: joseantonio.lopez@cchs.csic.es

2 Departamento de Biología Vegetal II, Facultad de Farmacia, Universidad Complutense. E-28040 Madrid. Spain. E-mail: dsmata@ucm.es, rgavilan@ucm.es
} 
populations of both pine species is heightened by their current fragmented distribution (Génova \& al., 2009), since predicted genetic and demographic consequences of habitat fragmentation might lead to a progressive loss of genetic diversity and to increasing differentiation among populations, lowering their evolutionary potential to respond to environmental changes (Benito Garzón \& al., 2006, 2008a, 2008b; Gea-Izquierdo \& al., 2015).

Black pine (Pinus nigra s.1.) is the most widely distributed pine species in high-altitude areas in the Mediterranean Basin; Pinus nigra subsp. salzmannii occurs in central and eastern Spain and southern France (Catalán \& al., 1991). In the Iberian Peninsula, Spanish black pine forests are mainly found in the supramediterranean bioclimatic belt under humid or sub-humid climates (900-1500 m asl), although the relict populations of the Spanish Central System on granitic rocks (Gredos and Guadarrama ranges) are also found under cold perhumid climate (Regato \& al., 1991, 1992, 1995; Regato \& Del Río, 2009). Scots pine (Pinus sylvestris s.1.) is a Eurosiberian forest tree species growing in a large variety of ecological conditions from Western Europe to Asia and the most widely spread among pine species (Ruby \& Wright, 1976). Its natural range extends northwards from warm southern areas of Spain and the mountains of northern $\operatorname{Iran}\left(37^{\circ} \mathrm{N}\right)$ to artic latitudes in Fennoscandia $\left(71^{\circ} \mathrm{N}\right)$, and westwards from longitudes $6^{\circ} \mathrm{W}$ (Scotland and Iberian Peninsula) to $135^{\circ} \mathrm{E}$ in Siberia (Boratynski, 1991). While most of its natural range is continuous, there are also large populations of Scots pine disconnected from the main natural range such as in the Iberian Peninsula, where currently this species inhabits disjunct mountainous areas (700-2600 m asl) with large distances between populations (Gil \& al., 1991; Alía \& al., 2001).

Geobotanical interpretation of high-mountain pine forests of the Gredos range have led to controversial interpretations and positions between those who accept their natural character and those who rule (Costa \& al., 1990; Alcalde \& al., 2006). This debate is still ongoing despite a growing body of evidence indicating the native origin of pine forests from historical and palaeoecological studies (Génova \& al., 1992, 2009). In the Gredos range, macrofossil and palynological records show the presence of both pine species throughout the Holocene (Rubiales \& al., 2007, 2012; López-Sáez \& al., 2014), suggesting the permanence of widespread high-mountain pine woodlands in these mountains at least since the mid-Holocene, as well as its progressive disappearance in the last two thousand years as a result of fires, logging and high pastoral pressure (López-Merino \& al., 2009; López-Sáez \& al., 2009, 2016a, 2016b).

Spanish black pine forests of the Gredos range are known for a long time (Gómez Manzaneque, 1988; Regato \& al., 1992), although no phytosociological approach has been done so far. Only Galán de Mera \& al. (1999) have conducted a first approximation of their communities, including them within the Vaccinio-Piceetea class, Pinetalia sylvestris order and Cytision oromediterranei alliance, but without describing any own phytosociological association of these forests. These facts sharply contrast with the rest of Spanish black pine forest communities in the Iberian Peninsula, for which various associations and subassociations have been described in the Pyrenees, the Iberian and Baetic ranges (Vigo, 1979; Gamisans \& Gruber, 1988; Valle \& al., 1988; Regato \& Escudero, 1989; Gamisans \& al., 1991; Regato \& al., 1995; Regato \& Elena Rosselló, 1995; RivasMartínez \& al., 2001, 2002, 2011). It is also noted that the high state of degradation of Pinus nigra subsp. salzmannii forests of the Gredos range has so far hindered any attempt to syntaxonomic description (Regato \& al., 1992; Regato \& Del Río, 2009). A similar situation has occurred with Scots pine forests of the Gredos range, although some communities throughout the Iberian Peninsula have been described in the Pyrenees, the northern and southern Iberian range, Baza and Sierra Nevada mountains in the Baetic range (RivasMartínez, 1987; Loidi \& al., 1997a, 1997b; Rivas-Martínez \& al., 2001, 2002). Although Pinus sylvestris forests from the Spanish Central System (P. sylvestris var. iberica) have hardly been treated into the phytosociological literature, with some exceptions for the Ayllón, Guadarrama and Pela ranges (De la Cruz \& Peinado, 1996; Rivas-Martínez \& al., 2001, 2002), recently, the combination of modern pollen studies and floristic relevés has helped to define two phytosociological associations comprising the Scots pine forests of the Gredos range (López-Sáez \& al., 2013). Nevertheless the geobotanical recognition of 
Scots pine forests as a series of vegetation in the Gredos mountains was highlighted by Rivas-Martínez \& al. (2011), for communities located on the supramediterranean and supratemperate (submediterranean) bioclimatic belt.

In this work, our aims are to (i) determine the plant communities of Pinus nigra Arnold subsp. salzmannii (Dunal) Franco and Pinus sylvestris L. var. iberica Svoboda forest vegetation in the Gredos range, and (ii) define their ecological and geographical gradients.

\section{Material and Methods}

\section{Study area}

The Gredos range is located at the western sector of the Spanish Central System, showing the highest altitudes $(>2000 \mathrm{~m}$ asl $)$ of the whole of this massif, which separates the basins of the Douro and Tagus rivers (Figure $1)$. This range is a chain of sunken and elevated blocks of granitic and metamorphic lithology, separated by intramontane valleys. The tectonic constitution, a tilted horst, with a fault orientated towards the south, imposes a clear asymmetry in both the northern and southern faces. The climate is of a Mediterranean type, dry and cold in winter $\left(0^{\circ} \mathrm{C}-2^{\circ} \mathrm{C}\right)$ and dry and warm $\left(20^{\circ} \mathrm{C}-22^{\circ} \mathrm{C}\right)$ in summer, with a summer drought period lasting 3-5 months and heavy rainfall in autumn and winter. Precipitation is on average $1400 \mathrm{~mm}$ per year. The study area belongs to the Bejaran-Gredensean sector, Carpetan-Leonese biogeogaphical subprovince, Iberian Western Mediterranean province, Western Mediterranean subregion, Mediterranean region (Rivas-Martínez \& al., 2007; Sánchez-Mata, 2015).

Figure 1. Location of the study area in the Gredos range (central Spain). For locating each relevé see the Material and methods section, Tables 4 to 6 .

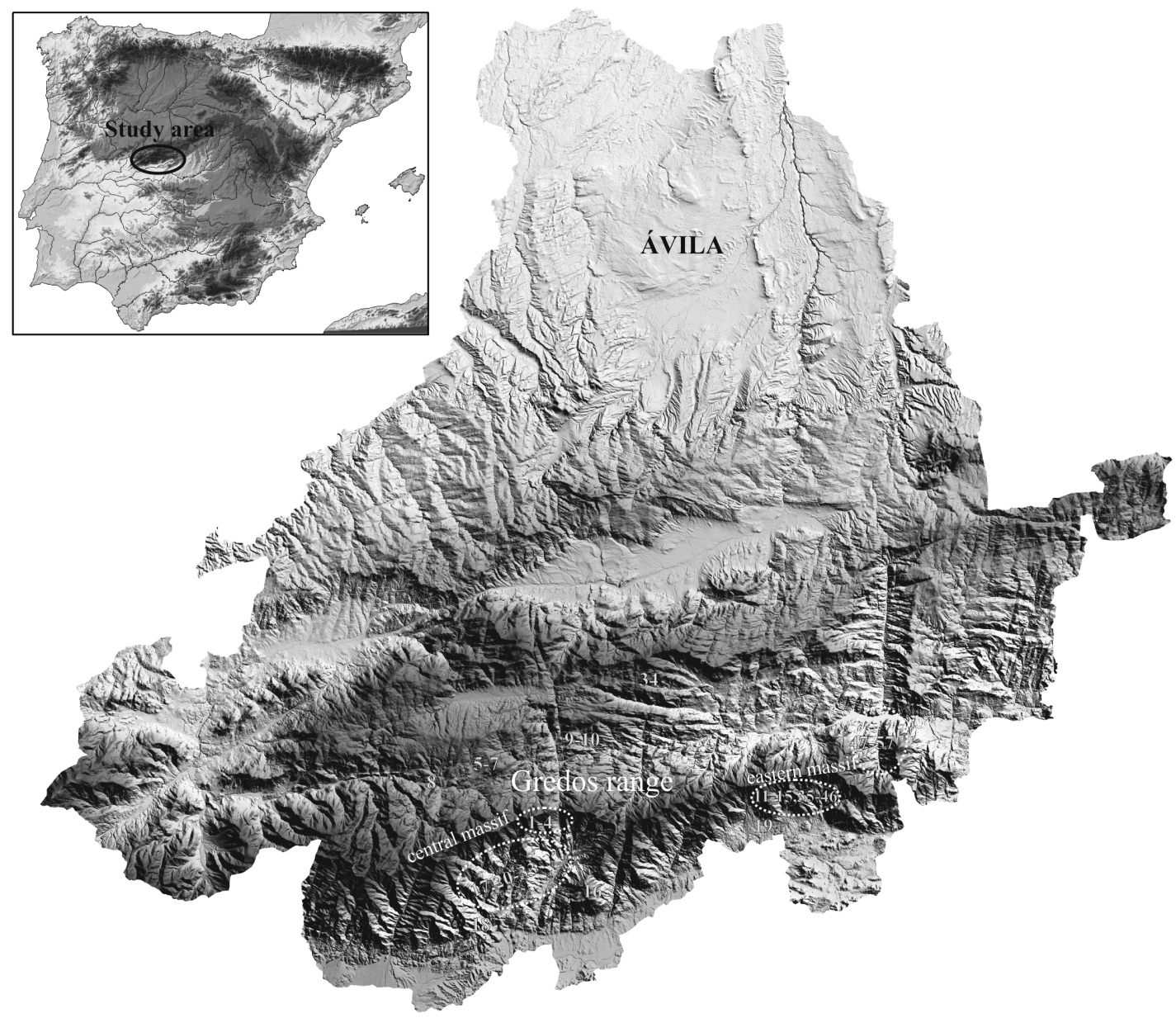


In Gredos range, Pinus sylvestris var. iberica forests are part of (sub)mediterranean pine forests (González Molina, 2006). This pine species appears very unevenly on the southern slopes of the central massif of the mountain and sporadically in the eastern one (Molina Moreno, 1992; Mancebo \& al., 1993), constituting, with some exception, extremely small isolated forests altered by the fire regime and pastoral pressure in the supra- and orosubmediterranean bioclimatic belts (López-Sáez \& al., 2009, 2014). However, on the northern slope of Gredos range, it covers an area of about 2000 ha in the upper basins of the Alberche and Tormes rivers, where it forms notable woodlands in Hoyos del Espino, Navarredonda and Hoyocasero in the supramediterranean and suprasubmediterranean bioclimatic belts (Génova \& al., 2009; Sánchez-Mata, 2015). In addition to the pure forests, it also forms mixed forests with Pinus nigra subsp. salzmannii depending on ecological gradients (López-Sáez \& López-García, 1994; Regato \& al., 1995).

Spanish black pine populations of the Spanish Central System are unique to peninsular level for being the only ones exclusively developed on siliceous substrates (granitic rocks, gneisses and micaschists) (Génova \& Moya, 2012). Their populations in the Gredos range mark the western limit of the species throughout its range (Regato \& al., 1995). In these mountains, Pinus nigra subsp. salzmannii appears sparsely as isolated pine copses sometimes within maritime pine $(P$. pinaster s.1.) or Pyrenean oak (Quercus pyrenaica) forests- on the southern slopes of the central and eastern massifs (Regato \& al., 1992), although some isolated populations develop on the northern slopes in the Iruelas valley as well as a very important one (40 ha) in the municipality of Navalacruz (Gandía \& al., 2005; Arias \& al., 2011). Somehow, it can be said that this pine in the Gredos range has a rupicolous vocation, as in many other peninsular territories (Regato \& al., 1991), occupying rocky outcrops and steep slopes of little edaphic development.

\section{Data collection}

A total of 57 relevés comprehending different formations of Pinus nigra subsp. salzmannii and $P$. sylvestris var. iberica in the Gredos range were analysed (Figure 1). Forty nine were obtained via field surveys conducted from February 2010 until October 2015, covering the entire area and known locations of both species. The remaining relevés (8) were collected from earlier studies (López-Sáez \& al., 2013). The phytosociological approach (Braun-Blanquet, 1979) was used to obtain relevés, including topographic and other data of individual plots, such as altitude, longitude, latitude and aspect. Additionally alder (Alnus glutinosa), maritime pine (Pinus pinaster s.1.) and Pyrenean oak (Quercus pyrenaica) forests which locally include relict Spanish black pines were also sampled. We chose homogenous sampling plots with an area of $100 \mathrm{~m}^{2}$.

The location of each relevé is referred in Tables 4 and 5, except for the following relevés: 18, Arenas de San Pedro, Río Pelayos, $530 \mathrm{~m}$ asl; 19, Piedralaves, Monte Dehesa Avellaneda, $805 \mathrm{~m}$ asl; 23, El Arenal, Collado de la Vuelta, 1,175 $\mathrm{m}$ asl; 39, Piedralaves, Garganta de Serradilla, $1350 \mathrm{~m}$ asl; 41, Piedralaves, Robledo de la Angostura, $1240 \mathrm{~m}$ asl; 48, El Tiemblo, Arroyo del Puerto, $880 \mathrm{~m}$ asl; 49, El Tiemblo, Cerro del Moro, $915 \mathrm{~m}$ asl; 50, El Tiemblo, Casa de Las Lamos, 1040 $\mathrm{m}$ asl; and, 51, El Tiemblo, Choza Lumbrero, $1180 \mathrm{~m}$ asl.

\section{Statistical analyses}

In order to establish ecological, floristic and phytogeographical relationships among relevés we have carried out multivariate analyses based on classification and ordination of vegetation data.

Classification was performed by hierarchical cluster analysis (HCA) with the program IBM SPSS Statistics 21 using the matrix of the Euclidean distance and Ward's minimum variance method (Ward, 1963), which has been shown to optimize the internal homogeneity of clusters and to establish clearer dichotomies among them, as well as to classify continuous variables better (Wildi, 1989; Escudero \& al., 1994).

Detrended correspondence analysis (DCA) was performed using CANOCO 4.5 (ter Braak \& Šmilauer, 2002). Detrending by segments, square-root transformation of the cover abundances of taxa and downweighting of rare species were applied. Although the longest gradient is shorter than 3 S.D. (Table 1), the unimodal method is also considered as an appropri- 
ate analysis of community compositional data, even in the case of short environmental gradients (Lepš \& Šmilauer, 2003). Furthermore, in the DCA, several variables were used as passive explanatory ones aiming to support the interpretation of the ordination diagram. Correlations between DCA relevé scores and explanatory variables were analysed by Spearman's rank correlation with STATISTICA 9.1 software (http://www.statsoft.com; Table 2).

To define diagnostic species for each vegetation type, we used the phi coefficient $(\Phi)$ of fidelity (Chytrý \& al., 2002). As diagnostic species (Table 3 ), we used only species with $\Phi$ $\geq 0.25$ and a statistically significant $(p<0.01)$ association with a particular vegetation type according Fisher's exact test, performed in the JUICE 7.0 program (Tichý, 2002). If a taxon had a fidelity value higher than $25 \%$ in only one synoptic table, then it was considered as diagnostic of the vegetation unit of the hierarchical level represented by the corresponding synoptic table, whereas if the same value is given in more than one synoptic table, then it was considered as differential of the hierarchical level in which it presented the highest fidelity value.

Traditionally, the phi coefficient considers only the presence/absence of taxa, so that fidelity values calculated using this coefficient are not influenced by species cover or abundance (Tichý \& al., 2011). Its main advantage it its independence of dataset size (Tichý, 2002). The highest $\Phi$ value (1) is achieved if the species occurs in all relevés of the vegetation unit and is absent elsewhere. A positive value $<1$ mean that the species is absent from some relevés of the vegetation unit or is present in some relevés outside the vegetation unit. Furthermore, we used an additional criterion to screen off taxa with a smooth decrease of their percentage constancy (Table 3). A $\Phi$ value of 0 is obtained when the relative frequency of the species (constancy) in the vegetation unit equals the relative frequency in the rest of the dataset, thus indicating no relation between the target species and the target vegetation unit (Tichý \& Chytrý, 2006).

\section{Nomenclature}

Taxonomic nomenclature and authorities follow the published volumes of Flora iberica (Castroviejo \& al., 1986-2015) and the com- pilations of Flora Europaea (Tutin \& al., 1964-1980) for taxa not included in the former; the exceptions are specifically mentioned in the floristic appendix. Syntaxonomical scheme, nomenclature, and syntaxa authorities follow the compilations and proposals of Rivas-Martínez \& al. (2001, 2002, 2011).

\section{Results}

The 57 relevés (hereinafter samples) were classified by means of HCA into seven sample groups, which correspond to ecologically and phytogeographically interpretable vegetation units. Different levels of division were accepted. As shown by the dendrogram analysis (Figure 2), two main clusters emerged (A and B), corresponding to the segregation of the two main vegetation units compared in this work, where Spanish black pine and Scots pine forests in the Gredos range were separated. While three of these clusters represent the Pinus sylvestris var. iberica forests, the last four are formed by pure or mixed $P$. nigra subsp. salzmannii communities. Cluster A makes reference to Pinus sylvestris var. iberica woodlands and cluster B make reference to the remaining woodlands containing $P$. nigra subsp. salzmannii, with a truncation level of 26.25 .

Table 1. Results of Detrended Correspondence Analysis (DCA) of 57 relevés hosting 98 species.

\begin{tabular}{|l|l|l|}
\hline \multicolumn{1}{|c|}{ Axes } & \multicolumn{1}{c|}{ Axis 1 } & \multicolumn{1}{c|}{ Axis 2 } \\
\hline Eigenvalues & 0.131 & 0.067 \\
\hline Lengths of gradient & 1.013 & 1.060 \\
\hline $\begin{array}{l}\text { Cumulative percentage } \\
\text { variance of species data }\end{array}$ & 21.3 & 32.1 \\
\hline
\end{tabular}

Inside cluster A, we can detach, at a smaller range (17.10), the sub-clusters A1 and A2; respectively separating orotemperate submediterranean (oceanic) Scots pine communities (cluster A1) from those of the supratemperate supramediterranean (oceanic) ones (cluster A2). The following threshold of cluster A1 (truncation level of 11.15) clearly separates samples containing Spanish black pine (cluster A1b) from those without (cluster A1a). Relating to cluster B, the major segregation at truncation level 6.77, separates B1 and B2 clades. The main disjunction promoted here is due to the significant presence of other 
Figure 2. Hierarchical cluster analysis (HCA) of the 57 relevés of high-mountain pine forests of the Gredos range. Dotted lines indicate the different thresholds that subdivide the dendrogram mentioned in the text, from right to left.

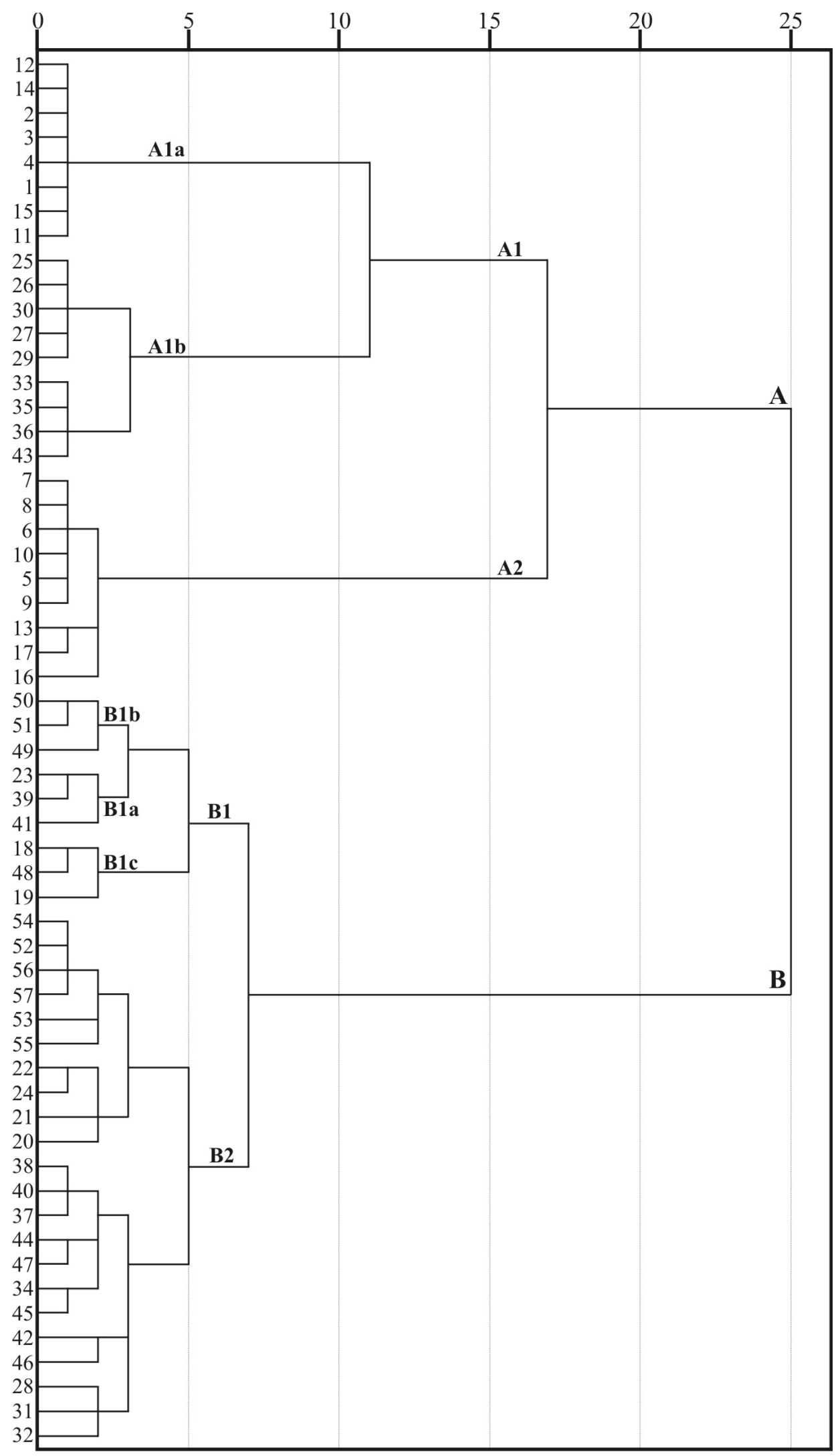


Figure 3. Detrended correspondence analysis (DCA-biplot) of the 57 investigated relevés (a) and selected species (b) in the Gredos range. Vegetation units representing the plant communities defined by cluster analysis (Figure 2) are marked by different symbols and framed. Explanatory variables describing the ecological background of the first two axes (displayed by arrows) were plotted a posteriori. We use 'short writting mode' for the following taxa: Carex elata subsp. reuteriana (C. reuteriana), Festuca elegans subsp. merinoi (F. merinoi), Festuca paniculata subsp. multispiculata (F. multispiculata), Juniperus communis subsp. alpina (J. alpina), Leucanthemopsis pallida subsp. alpina (L. alpina), and Potentilla alpina subsp. apiifolia (P. apiifolia).
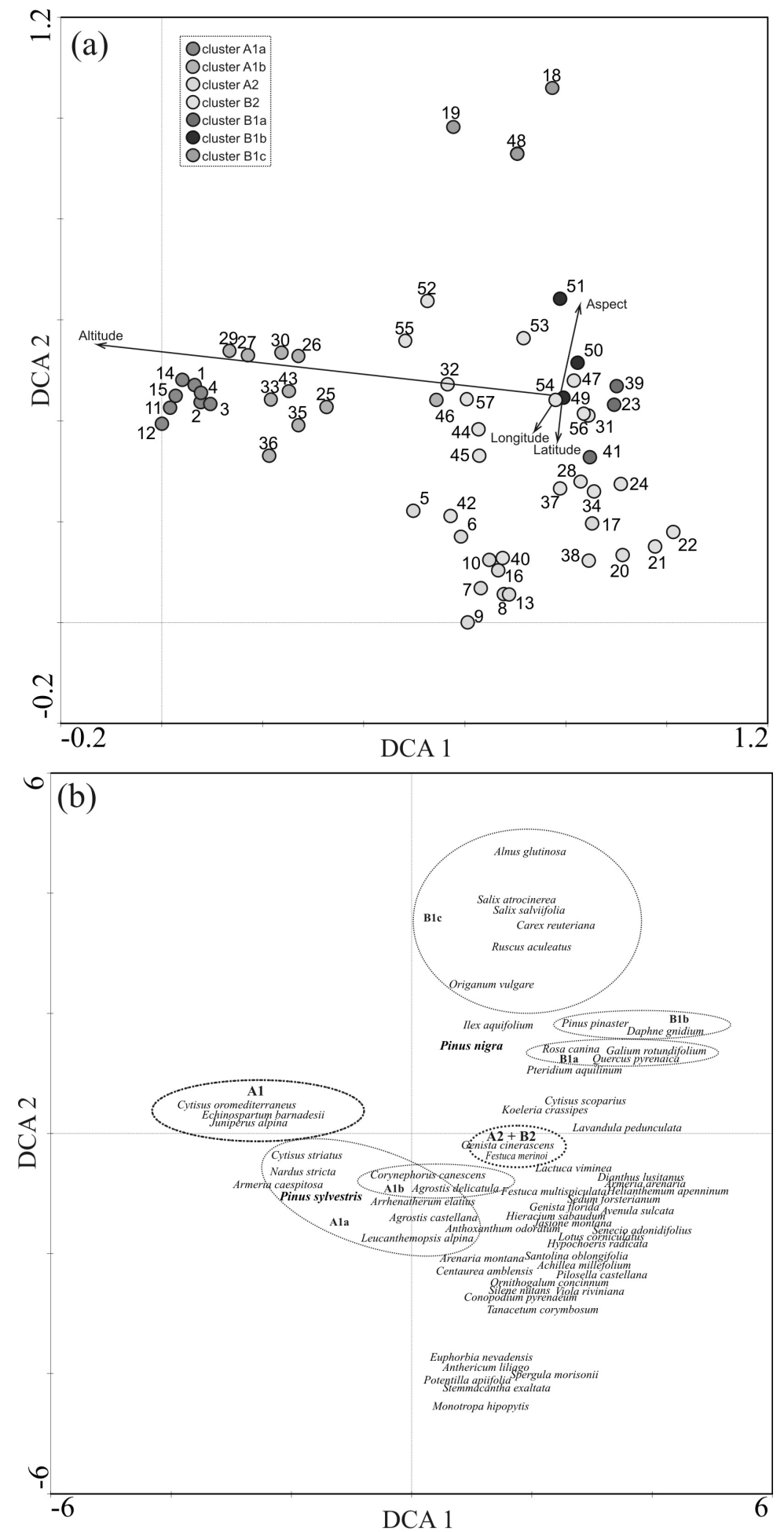
forest species in cluster B1, while the cluster B2 corresponds to pure Spanish black pine forests. The under clusters B1a to B1c, respectively segregate other forest formations that contain sporadically some Spanish black pines: Quercus pyrenaica woodlands (cluster B1a), Pinus pinaster s.1. forests (cluster B1b), and Alnus glutinosa riparian communities (cluster B1c).

In the ordination diagrams of Figure 3 the first two DCA axes were plotted. The eigenvalue of the first DCA axis was 0.131 and its length was 1.013 , while the corresponding values for the second DCA axis were respectively 0.067 and 1.060 (Table 1). Both DCA axes account for $32.1 \%$ of the cumulative percentage variance of species data (total inertia equal to 0.616 ).

Spearman rank correlation analysis between sample scores from the DCA analysis and the environmental variables (Table 2) revealed that altitude had the highest correlation with the sample sites (correlation with the first DCA axis: $r=-0.69, p<0.01$; Figure 3a). In fact, in the DCA ordination diagram of the relevés, there is a clear altitudinal gradient along the DCA axis 1: orosubmediterranean Pinus sylvestris var. iberica and $P$. nigra subsp. salzmannii dominated forests appear at the left part of the axis (clusters A1a and A1b), while suprasubmediterranean ones (clusters A2 and B2) take place through the right part of the axis respectively. Spearman rank correlation coefficient also revealed that altitude has a significant effect on vegetation along the axis 1 (Figure 3b): Pinus sylvestris var. iberica, Cytisus oromediterraneus, Echinospartum barnadesii, Nardus stricta and Juniperus communis subsp. alpina are located on the left side of the DCA axis 1; P. nigra subsp. salzmannii, Festuca elegans subsp. merinoi, and Genista cinerascens in the middle of it; and, finally, Quercus pyrenaica, P. pinaster s.1., Daphne gnidium and Lavandula pedunculata, among others, on the right part of DCA axis 1.

The second axis was weakly correlated with aspect $(r=0.18)$ and altitude $(r=0.14$; Table 2), which does not have a meaningful explanation on the vegetation differentiation. Along DCA axis 2, samples from cluster B1c (alder forests) were located at the top of the ordination diagram, clusters A1a, A1b, B2, $\mathrm{B} 1 \mathrm{a}$ and $\mathrm{B} 1 \mathrm{~b}$ in the middle, and cluster $\mathrm{A} 2$ with some samples from cluster B2 at the bottom (Figure 3a). Latitude and longitude were negatively correlated to DCA axis 2, although they are not significant variables in any of the two axes. In Figure $3 b$ the scores of the diagnostic taxa of cluster B1c (Alnus glutinosa, Salix atrocinerea, S. salviifolia, Carex elata subsp. reuteriana, Ruscus aculeatus and Origanum vulgare) are plotted at the top of DCA axis 2, probably indicating that this axis may have some relationship to environmental or soil moisture.

Table 2. Spearman rank correlations coefficients $(p<0.01)$ between the sample scores on the DCA axes 1 and 2 and environmental variables.

\begin{tabular}{|l|c|c|}
\hline Variable & Axis 1 & Axis 2 \\
\hline Altitude & -0.69 & 0.14 \\
\hline Aspect & 0.04 & 0.18 \\
\hline Latitude & 0.003 & -0.07 \\
\hline Longitude & -0.007 & -0.06 \\
\hline
\end{tabular}

The classification of the seven clusters, with statistically determined diagnostic species for each cluster, is presented in Table 3.

\section{Discussion and Conclusions}

In accordance with the classification analysis (Figure 2), it seen that the high-mountain forest vegetation in the Gredos range includes seven different plant communities. Two of them are dominated by Pinus nigra subsp. salzmannii (clusters $\mathrm{A} 1 \mathrm{~b}$ and $\mathrm{B} 2$ ), one of which (cluster A1b) is codominated by $P$. sylvestris var. iberica. Pure Scots pine forests comprise clusters A1a and A2, which are separated in the DCA ordination diagram along an altitudinal gradient in the DCA axis 1 (Figure 3a). Finally, cluster B1 represents forest formations dominated by other tree species Quercus pyrenaica (B1a), Pinus pinaster s.l. (B1b) and Alnus glutinosa (B1c)- that can promptly contain Spanish black pines $(P$. nigra subsp. salzmannii). The main factor affecting the floristic differences between communities in the Gredos range is altitude, which is signiticantly correlated with DCA axis 1 (Table 2), reflecting, among others, a temperature and precipitation gradient.

Cluster A1a represents xerophilous, orotemperate submediterranean (orosubmediterranean) hyperhumid relict Scots pine mesoforests from the middle oromediterranean belt of the Gredos range ( 1600-1800 $\mathrm{m}$ asl). Relevés from cluster A1a scored 
Table 3. Synoptic table of 57 relevés from high-mountain pine forests of the Gredos range, based on fidelity phi coefficient ( $\Phi$ x 100) and percentage constancy of species for each cluster (relevé groups). Fidelity was computed using the phi coefficient applied to a data set with the size of vegetation units (clusters A and B) equalized to $0.15 \%$ of the size of the entire data set. Diagnostic species (values in bold) are those with $\Phi \geq 25$; they are ranked by a decreasing value of $\Phi$. Dashes indicate negative fidelity or positive but non-significant fidelity at $p<0.01$. Superscript values indicate percentage frequency (constancy) of species; dots indicate species absence. Only species with a constancy $>25 \%$ are listed.

\begin{tabular}{|c|c|c|c|c|c|c|c|c|c|c|c|c|c|c|}
\hline Cluster & \multicolumn{2}{|c|}{ Ala } & \multicolumn{2}{|c|}{$\mathrm{A} 1 \mathrm{~b}$} & \multicolumn{2}{|c|}{$\mathrm{A} 2$} & \multicolumn{2}{|c|}{$\mathrm{B} 2$} & \multicolumn{2}{|c|}{ B1a } & \multicolumn{2}{|c|}{$\mathrm{B} 1 \mathrm{~b}$} & \multicolumn{2}{|c|}{$\mathrm{B} 1 \mathrm{c}$} \\
\hline Number of relevés & \multicolumn{2}{|c|}{8} & \multicolumn{2}{|c|}{10} & \multicolumn{2}{|c|}{9} & \multicolumn{2}{|c|}{21} & \multicolumn{2}{|c|}{3} & \multicolumn{2}{|c|}{3} & \multicolumn{2}{|c|}{3} \\
\hline Diagnostic species of cluster A: & & & & & & & & & & & & & & \\
\hline Pinus sylvestris var. iberica & 43.5 & 100 & 44.5 & 100 & 44.5 & 100 & $\begin{array}{ll}-- \\
-\end{array}$ & 5 & -- & . & -- & . & -- & . \\
\hline \multicolumn{15}{|l|}{ Diagnostic species of cluster A1: } \\
\hline Echinospartum barnadesii & 56 & 100 & 57.6 & 100 & --- & . & --- & 14 & --- & . & --- & . & --- & . \\
\hline Juniperus communis subsp. alpina & 51.8 & 88 & 65.2 & 100 & -- & . & -- & 5 & -- & . & -- & . & -- & . \\
\hline Cytisus oromediterraneus & 40.7 & 100 & 41.4 & 100 & --- & 78 & --- & 23 & -- & . & -- & . & -- & . \\
\hline \multicolumn{15}{|l|}{ Diagnostic species of cluster A1a: } \\
\hline Cytisus striatus subsp. eriocarpus & 92.5 & 88 & -- & . & --- & . & +- & . & -- & . & -- & . & -- & . \\
\hline Armeria caespitosa & 75.2 & 100 & -- & 11 & --- & . & $-\cdots$ & 18 & -- & . & -- & . & -- & . \\
\hline Nardus stricta & 47 & 25 & -- & $\cdot$ & -- & . & -- & $\cdot$ & -- & . & --- & . & --- & . \\
\hline $\begin{array}{l}\text { Leucanthemopsis pallida subsp. } \\
\text { alpina }\end{array}$ & 40.6 & 75 & --- & 11 & --- & 56 & --- & 14 & --- & 33 & --- & 33 & --- & . \\
\hline Arrhenatherum elatius s.1. & 36.5 & 100 & -- & 33 & -- & 56 & $-\cdots$ & 68 & -- & . & --- & 33 & -- & . \\
\hline Agrostis castellana & 27 & 100 & --- & 11 & --- & 44 & 24.7 & 91 & --- & 100 & --- & 67 & --- & 67 \\
\hline \multicolumn{15}{|l|}{ Diagnostic species of cluster A1b: } \\
\hline Corynephorus canescens & --- & . & 39.1 & 56 & -- & 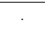 & -- & 27 & --- & . & --- & 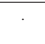 & --- & . \\
\hline Agrostis delicatula & --- & . & 30.5 & 78 & --- & . & 26.3 & 64 & -- & 67 & --- & 33 & -- & . \\
\hline \multicolumn{15}{|l|}{ Diagnostic species of cluster A2: } \\
\hline Senecio adonidifolius & --- & $\cdot$ & --- & $\cdot$ & 63.7 & 44 & -- & $\cdot$ & --- & . & --- & . & --- & . \\
\hline Hieracium sabaudum & --- & . & --- & . & 63.7 & 44 & --- & . & -- & 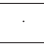 & --- & . & --- & . \\
\hline Spergula morisonii & --- & . & -- & . & 63.3 & 56 & --- & 5 & --- & . & --- & $\cdot$ & --- & . \\
\hline Lotus corniculatus s.1. & --- & . & --- & . & $\mathbf{5 7 . 5}$ & 89 & -- & 23 & --- & 67 & -- & 33 & --- & . \\
\hline Santolina oblongifolia & --- & . & --- & 11 & 55.8 & 78 & -- & 23 & --- & . & --- & . & --- & . \\
\hline Anthericum liliago & --- & . & --- & . & 54.7 & 33 & --- & . & --- & . & --- & . & --- & . \\
\hline Achillea millefolium & --- & $\cdot$ & -- & $\cdot$ & 53.4 & 100 & -- & 45 & -- & 33 & -- & 67 & --- & . \\
\hline Genista florida & --- & . & --- & . & 53.4 & 100 & --- & 41 & --- & 100 & --- & 33 & --- & . \\
\hline Hypochoeris radicata & --- & . & -- & . & 49.5 & 100 & 20.5 & 59 & -- & 67 & -- & $\cdot$ & -- & . \\
\hline Conopodium pyrenaeum & -- & . & -- & . & 46.5 & 56 & -- & 9 & -- & 33 & -- & 33 & --- & . \\
\hline Silene nutans & --- & . & --- & 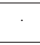 & 45.6 & 89 & -- & 50 & -- & 33 & --- & 33 & --- & . \\
\hline Viola riviniana & --- & $\cdot$ & -- & $\cdot$ & 44.4 & 67 & -- & 32 & -- & . & --- & $\cdot$ & --- & . \\
\hline Ornithogalum concinnum & -- & . & -- & $\cdot$ & 44.4 & 67 & --- & 27 & -- & 33 & -- & . & --- & . \\
\hline Ilex aquifolium & --- & . & --- & 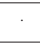 & 44.2 & 22 & --- & . & --- & . & --- & . & --- & . \\
\hline Stemmacantha exaltata & -- & . & -- & . & 44.2 & 22 & -- & . & -- & . & -- & 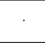 & -- & . \\
\hline Monotropa hypopytis & -- & $\cdot$ & -- & . & 44.2 & 22 & $-\cdots$ & . & -- & . & -- & . & -- & . \\
\hline Centaurea amblensis & --- & . & --- & . & 44.2 & 22 & 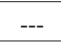 & . & --- & . & --- & . & --- & . \\
\hline Euphorbia nevadensis & -- & . & -- & . & 44.2 & 22 & -- & . & --- & . & -- & 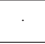 & -- & . \\
\hline Potentilla alpina subsp. apiifolia & --- & . & -- & . & 44.2 & 22 & $-\cdots$ & . & -- & . & -- & . & -- & . \\
\hline Tanacetum corymbosum & --- & . & --- & . & 44.2 & 22 & --- & . & --- & . & --- & . & --- & . \\
\hline Senecio jacobaea & -- & . & -- & . & 44.2 & 22 & -- & . & -- & . & -- & . & --- & . \\
\hline Pilosella castellana & -- & . & --- & . & 43.7 & 89 & -- & 50 & --- & 67 & --- & 33 & --- & 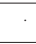 \\
\hline Arenaria montana & --- & . & --- & 44 & 43.7 & 89 & -- & 45 & --- & . & --- & . & --- & . \\
\hline Anthoxanthum odoratum & --- & . & --- & 33 & 41.8 & 89 & --- & 45 & -- & 33 & -- & 33 & --- & . \\
\hline Cytisus scoparius & -- & . & --- & . & 37.2 & 100 & -- & 68 & -- & 100 & -- & 100 & -- & 67 \\
\hline Jasione montana subsp. echinata & --- & . & -- & 22 & 32 & 89 & 25.6 & 73 & -- & 100 & --- & . & --- & . \\
\hline Pteridium aquilinum & --- & 50 & --- & 11 & 27.4 & 100 & -- & 82 & --- & 100 & -- & 100 & --- & 67 \\
\hline Diagnostic species of cluster B2: & & & & & & & & & & & & & & \\
\hline Lactuca viminea s.1. & --- & . & -- & 11 & -- & . & 56.2 & 50 & -- & . & -- & . & --- & 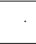 \\
\hline
\end{tabular}




\begin{tabular}{|c|c|c|c|c|c|c|c|c|c|c|c|c|c|c|}
\hline Helianthemum apenninum s.l. & --- & · & --- & & --- & & 53.3 & 32 & --- & & --- & & --- & . \\
\hline Avenula sulcata & --- & · & -- & 33 & -- & . & 47.1 & 64 & -- & 33 & -- & . & -- & . \\
\hline Lavandula pedunculata & -- & . & -- & . & -- & 11 & 47.1 & 64 & -- & 67 & -- & 33 & --- & . \\
\hline Dianthus lusitanus & --- & · & --- & & --- & & 44.3 & 36 & --- & & --- & 33 & --- & . \\
\hline Genista cinerascens & -- & . & -- & 22 & 28.5 & 100 & 36.8 & 100 & -- & 100 & -- & 100 & --- & . \\
\hline Pinus nigra subsp. salzmannii & -- & . & 27.4 & 100 & -- & . & 35 & 100 & -- & 100 & -- & 100 & -- & 100 \\
\hline Sedum forsterianum & -- & . & -- & 11 & -- & . & 31.5 & 32 & -- & 33 & -- & . & --- & . \\
\hline Armeria arenaria & -- & . & -- & . & -- & . & 30.2 & 23 & -- & 33 & -- & . & --- & . \\
\hline Koeleria crassipes & -- & . & --- & 33 & --- & . & 28.2 & 50 & --- & 33 & -- & 67 & -- & . \\
\hline $\begin{array}{l}\text { Festuca paniculata subsp. } \\
\text { multispiculata }\end{array}$ & --- & · & --- & 44 & --- & 67 & 26.6 & 77 & -- & 100 & --- & 33 & --- & . \\
\hline Festuca elegans subsp. merinoi & -- & 25 & -- & 33 & 25.1 & 100 & 26.4 & 95 & -- & 100 & -- & 100 & -- & 33 \\
\hline Pilosella pseudopilosella & -- & . & --- & 44 & -- & . & 24.5 & 41 & -- & 33 & --- & . & --- & . \\
\hline Clinopodium vulgare & -- & . & -- & 44 & -- & . & 20.8 & 41 & -- & 33 & -- & 33 & -- & . \\
\hline Rubus ulmifolius & --- & . & -- & 11 & -- & 11 & 19.7 & 55 & --- & 100 & --- & 67 & -- & 100 \\
\hline \multicolumn{15}{|l|}{ Diagnostic species of cluster B1a: } \\
\hline Quercus pyrenaica & -- & . & -- & . & -- & . & -- & 23 & 73.6 & 100 & -- & 33 & -- & . \\
\hline Galium rotundifolium & --- & . & --- & . & --- & . & --- & 55 & 54.5 & 100 & --- & 67 & -- & . \\
\hline Rosa canina & -- & . & -- & . & -- & 11 & -- & 18 & 49.9 & 67 & -- & 33 & -- & . \\
\hline \multicolumn{15}{|l|}{ Diagnostic species of cluster B1b: } \\
\hline Pinus pinaster s.1. & -- & 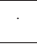 & -- & . & -- & . & -- & 55 & -- & 67 & 49.3 & 100 & -- & 33 \\
\hline Daphne gnidium & --- & . & --- & . & -- & . & -- & 18 & -- & 67 & 49.9 & 67 & --- & . \\
\hline \multicolumn{15}{|l|}{ Diagnostic species of cluster B1c: } \\
\hline Alnus glutinosa & -- & $\cdot$ & -- & . & -- & . & -- & . & -- & . & -- & . & 100 & 100 \\
\hline Salix atrocinerea & -- & $\cdot$ & --- & . & -- & 11 & -- & . & -- & . & +-- & . & 94.2 & 100 \\
\hline Salix salviifolia & --- & $\cdot$ & --- & . & -- & 11 & -- & . & -- & . & -- & . & 94.2 & 100 \\
\hline Carex elata subsp. reuteriana & -- & . & --- & . & -- & 11 & -- & 5 & -- & . & --- & . & 89.1 & 100 \\
\hline Ruscus aculeatus & --- & $\cdot$ & --- & . & -- & 11 & -- & 5 & -- & . & --- & 33 & 84.6 & 100 \\
\hline Origanum vulgare & --- & . & --- & 11 & --- & . & --- & 9 & --- & . & --- & . & 61.4 & 67 \\
\hline
\end{tabular}

chieftly between -0.11-0.12 S.D. units along DCA axis 1 (Figure 3a), being forest communities emplaced at higher elevations of the study. Diagnostic species of the community are Pinus sylvestris var. iberica, Juniperus communis subsp. alpina, Cytisus oromeiterraneus, C. striatus subsp. eriocarpus, Armeria caespitosa, Nardus stricta, Leucanthemopsis pallida subsp. alpina, Arrhenatherum elatius s.1. and Agrostis castellana (Figure 3b, Tables 3 and 4), as well as some western endemic element such as Echinospartum barnadesii (Sánchez-Mata, 1989, 1999; De la Fuente \& Ortúñez, 2001). This cluster corresponds to the Echinosparto barnadesii-Pinetum ibericae community described by López-Sáez \& al. (2013), representing the Scots pine forests of the timberline (Sánchez-Mata, 1999, 2015). These forests are considered as xerophilous because they are covered by winter snow for at least 4-5 months and grow on moderate to highly stony soils with low edaphic development (Gavilán \& al., 1998; Martínez-García \& Montero, 2000; Gavilán, 2005; López-Leiva \& al., 2009). In the Gredos range, this com- munity is only represented on the southern slope by isolated and scattered small forests or copses mostly affected by fires and grazing from prehistory (López-Sáez \& al., 2014, 2016a, 2016b). López-Sáez \& al. (2013) presented four relevés, southeast oriented, in the surrounding summits of Puerto del Pico Pass (La Rubía) from the central massif of the Gredos Range (1710-1800 $\mathrm{m}$ asl). In this paper four new inventories from the eastern massif of these mountains are presented (Table 4). Two come from Cerro de Pinosequillo in the municipality of Sotillo de la Adrada (1700$1710 \mathrm{~m}$ asl), and two more from Cabeza Pozo (Piedralaves; 1340-1400 $\mathrm{m}$ asl). In the eastern massif of the Gredos range this community appears at lower altitude than in the central one (even below $1400 \mathrm{~m}$ asl), compensating their situation with a predominant western orientation more influenced by the humid winds from the Atlantic Ocean (Gavilán \& al., 1998; Gavilán, 2005).

As a result of multivariate analyses (HCA and DCA), orosubmediterranean Pinus nigra subsp. salzmanni forest communities from the 
Table 4. Echinosparto barnadesii-Pinetum ibericae Sánchez-Mata, Gavilán \& López-Sáez in López-Sáez \& al. 2013 typicum (1-8); pinetosum salzmannii subass. nova (9-18)

(Avenello ibericae-Pinion ibericae, Pino sylvestris-Juniperetalia sabinae, Pino-Juniperetea)

\begin{tabular}{|c|c|c|c|c|c|c|c|c|c|c|c|c|c|c|c|c|c|c|}
\hline Altitude $(1=10 \mathrm{~m})$ & 177 & 180 & 175 & 171 & 140 & 135 & 170 & 1712 & 148 & 143 & 154 & 157 & 154 & 157 & 141 & 165 & 149 & 146 \\
\hline Aspect & $\mathrm{SE}$ & SE & SE & SE & NW & NW & $\mathrm{W}$ & SW & SE & SE & SE & SE & $\mathrm{SE}$ & SE & SE & $\mathrm{SE}$ & SE & SW \\
\hline Sample number (Fig. 3) & 1 & 2 & 3 & 4 & 11 & 12 & 14 & 15 & 25 & 26 & 27 & 29 & 30 & 33 & 35 & 36 & 43 & 46 \\
\hline Number of species & 15 & 15 & 17 & 20 & 12 & 15 & 14 & 14 & 17 & 15 & 11 & 11 & 13 & 16 & 22 & 22 & 17 & 28 \\
\hline Relevé number & 1 & 2 & 3 & 4 & 5 & 6 & 7 & 8 & 9 & 10 & 11 & 12 & 13 & 14 & 15 & 16 & 17 & 18 \\
\hline \multicolumn{19}{|l|}{ Characteristics } \\
\hline Pinus sylvestris var. iberica & 5 & 4 & 4 & 4 & 5 & 4 & 4 & 4 & 1 & 1 & 2 & 1 & + & 1 & 1 & 2 & 1 & 2 \\
\hline Cytisus oromediterraneus & 1 & 2 & 1 & 1 & 2 & 2 & 2 & 1 & 2 & 3 & 2 & 3 & 3 & 2 & 2 & 2 & 2 & 3 \\
\hline Echinospartum barnadesii & 2 & 3 & 3 & 4 & 3 & 3 & 3 & 2 & 1 & 1 & 2 & 2 & + & 2 & 3 & 2 & 2 & 1 \\
\hline $\begin{array}{l}\text { Juniperus communis subsp. } \\
\text { alpina }\end{array}$ & 1 & + & 1 & 2 & 2 & 1 & 1 & 2 & + & + & 1 & 2 & 1 & 2 & 1 & 2 & 1 & $\cdot$ \\
\hline Avenella iberica & 1 & 2 & + & + & 1 & 1 & 1 & 1 & 1 & 1 & 1 & 1 & + & . & + & 1 & 1 & 1 \\
\hline \multicolumn{19}{|l|}{ Differentials of typicum } \\
\hline Arrhenatherum elatius s.1. & 1 & 1 & + & + & 1 & 1 & 1 & 1 & . & · & . & . & . & . & + & 1 & + & 1 \\
\hline Agrostis castellana & 1 & + & 1 & 1 & 1 & 1 & 1 & 1 & . & . & . & . & . & . & . & . & 1 & 1 \\
\hline Armeria caespitosa & 1 & 1 & + & + & 1 & 1 & 1 & 1 & . & . & . & . & . & . & $\cdot$ & + & . & $\cdot$ \\
\hline Cytisus striatus subsp. eriocarpus & 1 & + & + & + & . & + & 1 & + & . & . & . & . & . & . & . & . & . & $\cdot$ \\
\hline $\begin{array}{l}\text { Leucanthemopsis pallida subsp. } \\
\text { alpina }\end{array}$ & . & + & + & + & + & + & . & + & . & . & . & . & . & $\cdot$ & $\cdot$ & + & . & $\cdot$ \\
\hline Nardus stricta & + & . & . & . & . & . & . & + & . & . & . & . & . & . & $\cdot$ & . & · & $\cdot$ \\
\hline \multicolumn{19}{|l|}{ Differentials of pinetosum salzmannii } \\
\hline Pinus nigra subsp. salzmannii & . & . & . & . & . & . & . & . & 3 & 3 & 3 & 3 & 2 & 2 & 3 & 2 & 3 & 2 \\
\hline Corynephorus canescens & . & . & . & . & . & . & . & . & 1 & + & 1 & 1 & 1 & + & + & 1 & + & 1 \\
\hline Agrostis delicatula & . & . & $\cdot$ & . & · & . & $\cdot$ & . & 2 & 1 & 2 & + & 1 & + & + & 1 & + & + \\
\hline \multicolumn{19}{|c|}{ Shrubby, forest and perennial companions } \\
\hline Festuca elegans subsp. merinoi & . & . & + & + & . & . & . & . & 1 & + & . & . & 1 & . & . & . & . & 3 \\
\hline $\begin{array}{l}\text { Festuca paniculata subsp. } \\
\text { multispiculata }\end{array}$ & . & . & . & . & . & . & . & . & + & . & . & . & . & + & 1 & + & . & $\cdot$ \\
\hline Festuca gredensis & 1 & 1 & 1 & 1 & 1 & 1 & 1 & + & 1 & 2 & 2 & 2 & 1 & 1 & 1 & 1 & . & 2 \\
\hline Genista cinerascens & . & . & . & . & . & . & . & . & + & + & . & . & . & . & . & . & . & 2 \\
\hline Luzula lactea & 1 & 1 & 1 & + & 1 & + & 1 & 1 & + & . & . & . & . & + & 1 & 1 & . & 1 \\
\hline Erica arborea & . & + & + & + & . & . & + & · & . & . & 1 & . & 1 & . & + & . & · & 1 \\
\hline Pteridium aquilinum & . & · & + & + & + & . & + & . & . & . & . & . & · & . & $\cdot$ & $\cdot$ & 1 & 2 \\
\hline Poa bulbosa & . & + & + & . & . & + & . & + & . & . & . & . & . & + & + & 1 & 1 & 1 \\
\hline Santolina oblongifolia & . & . & . & . & . & . & . & . & + & . & . & . & . & . & $\cdot$ & . & . & + \\
\hline Rubus ulmifolius & . & . & . & . & . & . & . & . & . & . & . & . & . & . & + & . & . & + \\
\hline Thymus mastichina & + & . & . & . & . & + & . & . & . & . & . & . & . & . & $\cdot$ & . & . & · \\
\hline \multicolumn{19}{|l|}{ Others } \\
\hline Cerastium ramosissimum & . & + & 1 & + & . & 1 & . & . & . & . & . & . & . & . & + & + & 1 & . \\
\hline $\begin{array}{l}\text { Rumex acetosella subsp. } \\
\text { angiocarpus }\end{array}$ & + & . & . & + & . & . & . & . & . & . & . & . & . & . & + & . & . & 1 \\
\hline Carduus carpetanus & + & . & . & . & . & . & . & . & . & . & + & . & + & . & $\cdot$ & + & . & 1 \\
\hline Jasione montana subsp. echinata & . & . & . & . & . & . & . & . & + & . & . & + & . & . & . & . & . & . \\
\hline Anthoxanthum odoratum & . & . & . & . & . & . & . & . & . & . & . & . & . & . & + & + & 1 & + \\
\hline Arenaria montana & . & & . & . & . & . & . & . & . & . & . & . & . & + & + & + & 1 & $\cdot$ \\
\hline Avenula sulcata & . & . &. & . &. &. &. &. & . & . &. &. & $\cdot$ & + & + & + &. & $\cdot$ \\
\hline
\end{tabular}

Other species: shrubby, forest and perennials companions: Sorbus aucuparia + in 4; Cytisus scoparius 1, Crataegus monogyna 1 and Pinus pinaster s.1. 1 in 46. Others: Jasione sessiliflora and Urtica dioica + in 4; Jasione montana subsp. echinata + in 25; Lactuca viminea s.1. and Origanum vulgare + in 26; Sedum forsterianum + in 33; Galium rotundifolium, Armeria arenaria and Silene nutans + in 46.

Localities: Ávila, Gredos range. 1-4: Cuevas del Valle, surrounding summits of 'Puerto del Pico' and 'La Rubia'; 56: Piedralaves, Cabeza Pozo; 7-8: Sotillo de la Adrada, Cerro de Pinosequillo; 9: El Arenal, Arroyo de los Torneros; holotypus subass.; 10: El Arenal, Arroyo de la Cabrilla; 11: El Arenal, Cerro de Coladillas; 12-13: El Arenal, Arroyo del Andrinal; 14: El Arenal, Risco de las Morillas; 15: Casavieja, Portacho del Alcaraván; 16: Casavieja, Garganta de la Chorrera; 17: Piedralaves, Peña Horcada; 18: Sotillo de la Adrada, Venero de las Apreturas. 
Gredos range (cluster A1b) are very well discriminated from samples of cluster A1a, corresponding, as noted earlier, to pure Scots pine forests communities (Figures 2 and 3 ). The bulk of relevés is placed in the left of the DCA diagram (0.15-0.33 S.D. units along DCA axis 1 ), although to the right of samples from cluster A la considering that relevés from cluster A1b are at lower altitudes (1410-1650 m asl; Figure 3a, Table 4). Samples from cluster A1b always include in their relevés Pinus sylvestris var. iberica (Table 4), representing mixed formations today dominated by $P$. nigra although in the past they could dominate both species (Regato \& al., 1991, 1992; López-Sáez \& al., 2014). Pinus sylvestris var. iberica, Echinospartum barnadesii, Juniperus communis subsp. alpina and Cytisus oromediterraneus are diagnostic species of both clusters Ala and Alb; while Corynephorus canescens, Agrostis delicatula and P. nigra subsp. salzmannii are the specific diagnostic species of cluster A1b (Figure 3b, Table 3).

The observed variability within cluster A1 is due to an altitudinal gradient. Pinus nigra subsp. salzmannii which appears to be more droughtresistant that $P$. sylvestris var. iberica (Aussenac, 1980), is a frequent co-dominant canopy tree only in lower sites (Figure 1). Given that forest types within clusters A1a and A1b share common species with similar ecology and, especially, migration histories during the late glacial and post-glacial periods (Génova \& al., 2009; LópezSáez \& al., 2013, 2014; Abel-Schaad \& al., 2014), all previously mentioned facts allow us to propose a new pinewood subassociation: Echinosparto barnadesii-Pinetum ibericae Sánchez-Mata, Gavilán \& López-Sáez in López-Sáez \& al. 2013 pinetosum salzmannii subass. nova hoc loco (Table 4, holotypus, relevé 9). It represents orotemperate submediterranean (orosubmediterranean) humid relict Spanish black pine mesoforests growing throughout the central and eastern Gredos Mountains (Figure 1, Table 4). As typical subassociation (typicum, cluster A1a), this new described subassociation is also represented on the southern slope of the Gredos range by isolated copses that have also suffered recurrent action of fires, overgrazing and indiscriminate felling (Regato \& al., 1992; Rubiales \& al., 2007, 2012; López-Sáez \& al., 2014). The ten inventories presented in this paper are always from southeast oriented populations (Table 4), because the Spanish black pine is a species that demand bright situations (Elena Rosselló \& al., 1985; Elena Rosselló \& Sánchez Palomares, 1991; Regato \& Elena Rosselló, 1995).

Cluster A2 included samples from xeromesophilous Pinus sylvestris var. iberica forest communities, mainly from the northern Gredos range (samples 5-10) but also from the southern slope (samples 13, 16, 17), developing at an altitudinal level ranging from 1300 to $1570 \mathrm{~m}$ asl on granite soils (Figure 1). The dominance of Pinus sylvestris var. iberica in their relevés places within the cluster A, differing from sub-cluster A1 by an altitudinal gradient (Figure 2). This group includes 9 relevés situated at the right of the DCA axis 1 (Figure 3a). These were classified by LópezSáez \& al. (2013) as Festuco merinoi-Pinetum ibericae from relevés only on the north side; although in this paper several on the southern slope of the Gredos range are presented (Table 5). Floristically, theses forests represent Pinus sylvestris var. iberica relict mesoforests from the supratemperate submediterranean and upper supramediterranean humid belt of the Gredos mountains, present both in the northern (Navarredonda de Gredos, Hoyos del Espino, Hoyocasero) and southern (La Adrada, San Esteban del Valle, Guisando) slopes in scattered nuclei. They are characterized by diagnostic species such as Senecio adonidifolius, Hieracium sabaudum and Spergula morisonii (Figure 3b, Table 3), among others, as well as dense broom communities mainly composed by Cytisus scoparius and Genista florida and by western endemic such as Festuca elegans subsp. merinoi, Centaurea amblensis, Ornithogalum concinnum and Genista cinerascens (MartínezGarcía \& Montero, 2000; De la Fuente \& Ortúñez, 2001). The relict population from sample 17 is located at $1170 \mathrm{~m}$ asl (supramediterranean thermotype areas), sheltered within a mountain stream and protected by a large granitic batholith which has allowed its survival at low altitude on the southern slope of the Gredos range.

Cluster B2 represents pure Spanish black pine forests in the lower supramediterranean humid bioclimatic belt of the Gredos range ( $\sim 900-1450 \mathrm{~m}$ asl), southeast oriented on the southern slope and northwest oriented on the northern slope of these mountains. This group includes 21 relevés situated at the right of the DCA axis 1 (Figure 3a). Diagnostic species of the community are Lactuca viminea s.l., 
Table 5. Festuco merinoi-Pinetum ibericae Sánchez-Mata, Gavilán \& López-Sáez in López-Sáez \& al. 2013 (Avenello ibericae-Pinion ibericae, Pino sylvestris-Juniperetalia sabinae, Pino-Juniperetea)

\begin{tabular}{|c|c|c|c|c|c|c|c|c|c|}
\hline Altitude $(1=10 \mathrm{~m})$ & 150 & 153 & 157 & 146 & 132 & 135 & 130 & 142 & 117 \\
\hline Number of species & 24 & 28 & 33 & 31 & 33 & 32 & 33 & 30 & 36 \\
\hline Relevé number & 1 & 2 & 3 & 4 & 5 & 6 & 7 & 8 & 9 \\
\hline Sample number (Figure 3) & 5 & 6 & 7 & 8 & 9 & 10 & 13 & 16 & 17 \\
\hline Aspect & SE & $\mathrm{SE}$ & SE & SE & $\mathrm{SE}$ & SE & $\mathrm{S}$ & SW & SE \\
\hline \multicolumn{10}{|l|}{ Characteristics } \\
\hline Pinus sylvestris var. iberica & 5 & 4 & 4 & 4 & 5 & 5 & 5 & 2 & 5 \\
\hline Genista cinerascens & 1 & 2 & 2 & 2 & 2 & 3 & 2 & 3 & 2 \\
\hline Festuca elegans subsp. merinoi & 2 & 2 & 2 & 2 & 3 & 2 & 3 & 3 & 3 \\
\hline Senecio adonidifolius & $\cdot$ & . & $\cdot$ & + & $\cdot$ & + & + & . & + \\
\hline Pilosella castellana & + & + & 1 & 1 & $\cdot$ & + & 1 & 1 & 1 \\
\hline Hieracium sabaudum & $\cdot$ & $\cdot$ & + & $\cdot$ & $\cdot$ & + & $\cdot$ & + & + \\
\hline Spergula morisonii & + & + & + & + & + & $\cdot$ & . & . & $\cdot$ \\
\hline Lotus corniculatus s.l. & . & + & 1 & 1 & 1 & + & + & 1 & 1 \\
\hline Santolina oblongifolia & + & + & + & 1 &. & + & 1 &. & 1 \\
\hline Anthericum liliago & $\cdot$ & $\cdot$ & $\cdot$ & $\cdot$ & + & + & + & . & $\cdot$ \\
\hline Achillea millefolium & + & + & + & 1 & 1 & + & + & 1 & 1 \\
\hline Silene nutans & + & + & + & 1 & + & $\cdot$ & 1 & 1 & 1 \\
\hline Hypochoeris radicata & + & 1 & + & 1 & 1 & + & + & 1 & 1 \\
\hline Genista florida & + & $\cdot$ & + & + & $\cdot$ & $\cdot$ & + & + & + \\
\hline Conopodium pyrenaeum & $\cdot$ & $\cdot$ & + & 1 & + & + & + & $\cdot$ & + \\
\hline Viola riviniana & . & + & + & $\cdot$ & $\cdot$ & + & + & 1 & 1 \\
\hline Ornithogalum concinnum & . & $\cdot$ & $\cdot$ & . & + & $\cdot$ & . & . & + \\
\hline Cytisus scoparius & + & + & 1 & 1 & 1 & + & 1 & 1 & + \\
\hline Pteridium aquilinum & + & 1 & + & 1 & 1 & + & 1 & 1 & 1 \\
\hline Jasione montana subsp. echinata &. & 1 & + & + & 1 & + & 1 & 1 & 1 \\
\hline Anthoxanthum odoratum &. & + & 1 & + & 1 & + & 1 & 1 & 1 \\
\hline Arenaria montana & . & + & + & + & + & + & $\cdot$ & $\cdot$ & $\cdot$ \\
\hline Euphorbia nevadensis & $\cdot$ & $\cdot$ & $\cdot$ & $\cdot$ & + & $\cdot$ & + & $\cdot$ & $\cdot$ \\
\hline Senecio jacobaea & $\cdot$ & $\cdot$ & $\cdot$ & $\cdot$ & 1 & + & $\cdot$ & $\cdot$ & $\cdot$ \\
\hline Ilex aquifolium & . & $\cdot$ & $\cdot$ & + & $\cdot$ & $\cdot$ & $\cdot$ & $\cdot$ & + \\
\hline \multicolumn{10}{|l|}{ Shrubby, forest and perennial companions } \\
\hline Cytisus oromediterraneus & 1 & 1 & 1 & + & + & $\cdot$ & + & + & $\cdot$ \\
\hline Erica arborea & 1 & 1 & 1 & 1 & 1 & 1 & 2 & 2 & 2 \\
\hline Festuca gredensis & + & + & + & + & 1 & + & + & 1 & 1 \\
\hline Luzula lactea & + & 1 & + & $\cdot$ & $\cdot$ & + & 1 & 1 & 2 \\
\hline Poa bulbosa & $\cdot$ & 1 & $\cdot$ &. & $\cdot$ & $\cdot$ & 1 & 1 & 1 \\
\hline Avenella iberica & 1 & 1 & 1 & 1 & + & 1 & 1 & 1 & 1 \\
\hline Agrostis castellana & + & + & 1 & 1 & 1 & + & 1 & + & 1 \\
\hline Festuca paniculata subsp. multispiculata & + & . & + & . & $\cdot$ & $\cdot$ & 1 & 1 & 1 \\
\hline Rosa canina & $\cdot$ & $\cdot$ & $\cdot$ & + & $\cdot$ & $\cdot$ & $\cdot$ & + & $\cdot$ \\
\hline \multicolumn{10}{|l|}{ Others } \\
\hline Arrhenatherum elatius s.l. & $\cdot$ & + & + & + & $\cdot$ & $\cdot$ & $\cdot$ & + & + \\
\hline Cerastium ramosissimum & $\cdot$ & 1 & 1 & 1 & $\cdot$ & 1 & 1 & + & 1 \\
\hline Rumex acetosella subsp. angiocarpus & + & + & + & + & $\cdot$ & + & 1 & 1 & 1 \\
\hline Carduus carpetanus & + & $\cdot$ & + & $\cdot$ & $\cdot$ & $\cdot$ & + & + & + \\
\hline Leucanthemopsis pallida subsp. alpina & + & + & 1 & 1 & $\cdot$ & + & 1 & 1 & 1 \\
\hline Aquilegia vulgaris & . & . & $\cdot$ & . & + & 1 & . & . & $\cdot$ \\
\hline Lychnis flos-cuculi & . & . & . & . & + &. & + & . & $\cdot$ \\
\hline Iris xiphium & $\cdot$ & $\cdot$ & $\cdot$ & $\cdot$ & + & + & $\cdot$ & $\cdot$ & $\cdot$ \\
\hline Arenaria querioides & $\cdot$ & $\cdot$ & + & $\cdot$ & + & $\cdot$ & $\cdot$ & $\cdot$ & $\cdot$ \\
\hline
\end{tabular}

Other species: shrubby, forest and perennials companions: Rubus corylifolius s.1. 1 in 9; Lavandula pedunculata 1, Frangula alnus, Crataegus monogyna and Sorbus aria + in 17. Others: Tanacetum corymbosum + in 8; Convallaria majalis, Caltha palustris, Polygonatum odoratum and Stemmacantha exaltata + in 9; Potentilla alpina subsp. apiifolia 1, Lilium martagon and Monotropa hypopytis + in 10.

Localities: Ávila, Gredos range. 1-3: Navarredonda de Gredos; 4: Hoyos del Espino; 5-6: Hoyocasero; 7: La Adrada, Pinara de la Virgen; 8: San Esteban del Valle, La Abantera; 9: Guisando, Chorreras de La Lancha. 
Helianthemum apenninum s.l., Avenula sulcata, Lavandula stoechas subsp. pedunculata, Dianthus lusitanus, Sedum forsterianum, Armeria arenaria, Koeleria crassipes and Festuca paniculata subsp. multispiculata (Figure 3b, Table 3). This community constitutes single cohort stand structure so that it was only dominated by Pinus nigra subsp. salzmannii in three layers and it is generally formed by mature stands in central and eastern massifs of the Gredos range (Regato \& al., 1991, 1992). These communities are mainly located in southeast exposures due to the thermic requirements of Spanish black pine (Figure 1), representing supratemperate submediterranean (suprasubmediterranean) humid relict forests. In fact, the Gredos range shows high phytoclimatic suitability for this species in areas with higher rainfall in winter and spring and cooler summer temperatures (Romera García \& al., 2001; García López \& al., 2005).
Although it is considered that the downweighting of fidelity values in relatively small relevé groups is desirable (Bruelheide, 2000), this fact could lead to a weaker differentiation of relatively rare vegetation units, especially in micro- or meso-scale phytosociological studies, as the presented here, and thus to the failure of their recognition. An additional improvement is the use of fidelity values of species following different hierarchical levels, as we proposed below. For instance, Pinus sylvestris var. iberica can be considered a transgressive diagnostic species having both diagnostic fidelity values in more than one group (clusters A1a, A1b, A2) and a constancy $>20 \%$ in all groups for which the species is diagnostic (Table 3) (Tichý \& Chytrý, 2006). The same circumstances can sign up for other species: Echinospartum barnadesii, Juniperus communis subsp. alpina and Cytisus oromediterraneus for clusters $\mathrm{A} 1 \mathrm{a}$ and $\mathrm{A} 1 \mathrm{~b}$; Agrostis delicatula for clusters A1b and B2; Hypochoeris radicata, Jasione montana subsp. echinata, Genista cinerascens and

Table 6. Genisto cinerascentis-Pinetum salzmannii Sánchez-Mata, Gavilán \& López-Sáez ass. nova (Avenello ibericae-Pinion ibericae, Pino sylvestris-Juniperetalia sabinae, Pino-Juniperetea)

\begin{tabular}{|c|c|c|c|c|c|c|c|c|c|c|c|c|c|c|c|c|c|c|c|c|c|}
\hline Altitude $(1=10 \mathrm{~m})$ & 125 & 105 & 110 & 120 & 125 & 127 & 135 & 113 & 143 & 135 & 134 & 144 & 135 & 140 & 92 & 149 & 125 & 115 & 152 & 123 & 123 \\
\hline Aspect & $\mathrm{SE}$ & SE & $\mathrm{SE}$ & $\mathrm{SE}$ & SE & SE & $\mathrm{SE}$ & $\mathrm{N}$ & $\mathrm{SE}$ & $\mathrm{SE}$ & $\mathrm{SE}$ & $\mathrm{SE}$ & NW & SE & NW & NW & NW & NW & NW & NW & SE \\
\hline Sample number (Figure 3) & 20 & 21 & 22 & 24 & 28 & 31 & 32 & 34 & 37 & 38 & 40 & 42 & 44 & 45 & 47 & 52 & 53 & 54 & 55 & 56 & 57 \\
\hline Number of species & 36 & 33 & 42 & 34 & 30 & 31 & 13 & 34 & 39 & 40 & 29 & 28 & 19 & 22 & 24 & 16 & 24 & 28 & 18 & 29 & 20 \\
\hline Relevé number & 1 & 2 & 3 & 4 & 5 & 6 & 7 & 8 & 9 & 10 & 11 & 12 & 13 & 14 & 15 & 16 & 17 & 18 & 19 & 20 & 21 \\
\hline \multicolumn{22}{|l|}{ Characteristics } \\
\hline Pinus nigra subsp. salzmannii & 4 & 3 & 3 & 3 & 3 & 2 & 3 & 4 & 2 & 3 & 2 & 3 & 3 & 4 & 3 & 3 & 2 & 3 & 3 & 3 & 3 \\
\hline Genista cinerascens & 1 & 2 & 2 & 3 & 3 & 3 & 3 & 2 & 1 & 2 & 2 & 3 & 3 & 1 & 2 & + & 1 & 1 & 1 & 2 & 1 \\
\hline $\begin{array}{l}\text { Festuca elegans subsp. } \\
\text { merinoi }\end{array}$ & 3 & 3 & 2 & 3 & 3 & 3 & 2 & 3 & 1 & 3 & 2 & 2 & 2 & 3 & 2 & 1 & + & 1 & . & 2 & + \\
\hline $\begin{array}{l}\text { Festuca paniculata subsp. } \\
\text { multispiculata }\end{array}$ & + & + & 1 & 1 & + & 1 & . & + & 1 & 1 & 1 & + & 1 & 1 & 1 & + & + & . & . & . & + \\
\hline Agrostis delicatula & 1 & 2 & 1 & 1 & . & 1 & 1 & 1 & 1 & + & 1 & 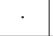 & . & . & . & . & . & 1 & 1 & 1 & $\cdot$ \\
\hline Hypochoeris radicata & 1 & 1 & 1 & 1 & . & . & + & 1 & + & 1 & 1 & . & . & . & . & . & + & + & . & 1 & + \\
\hline $\begin{array}{l}\text { Jasione montana subsp. } \\
\text { echinata }\end{array}$ & + & 1 & 1 & + & . & + & . & 1 & 1 & 1 & 1 & + & 1 & 1 & 1 & . & . & + & . & 1 & + \\
\hline Lactuca viminea s.1. & + & + & + & . & . & + & + & + & . & . & + & $\cdot$ & . & . & . & . & . & 1 & + & + & + \\
\hline Helianthemum apenninum s.1. & 3 & 1 & 1 & 1 & . & . & . & . & . & . & . & . & . & . & . & . & 1 & + & . & + & $\cdot$ \\
\hline Avenula sulcata & 1 & + & 1 & 1 & 1 & 1 & . & + & 1 & 1 & + & + & . & . & . & . & 1 & 1 & . & + & $\cdot$ \\
\hline Lavandula pedunculata & 2 & 1 & 1 & + & . & + & + & . & + & + & . & . & . & . & + & 1 & 2 & + & . & 1 & + \\
\hline Dianthus lusitanus & 1 & 3 & 1 & + & . & 1 & 1 & . & + & . & . & . & . & . & . & . & . & . & . & + & $\cdot$ \\
\hline Sedum forsterianum & 1 & 1 & 1 & + & + & . & . & . & . & + & . & · & . & . & . & . & . & . & + & . & $\cdot$ \\
\hline Armeria arenaria & 1 & + & 1 & + & . & . & . & . & . & . & . & . & . & . & . & . & . & . & . & . & $\cdot$ \\
\hline Koeleria crassipes & . & + & + & . & 1 & . & . & + & . & . & + & . & . & . & . & . & 1 & 1 & 1 & 1 & + \\
\hline \multicolumn{22}{|c|}{ Shrubby, forest and perennial companions } \\
\hline Cytisus oromediterraneus & . & . & . & . & . & . & . & . & . & . & . & 2 & . & . & . & 1 & 1 & . & 1 & . & $\cdot$ \\
\hline Festuca gredensis & 2 & 1 & 1 & 1 & 1 & 2 & + & 1 & 1 & 1 & + & 1 & 1 & 1 & 1 & 1 & 1 & 1 & 1 & 1 & 1 \\
\hline Echinospartum barnadesii & . & . & . & . & . & . & . & . & . & . & . & + & . & . & . & . & . & . & + & . & $\cdot$ \\
\hline Luzula lactea & 1 & 1 & 1 & 1 & + & 1 & . & 1 & 1 & 1 & 1 & 1 & 1 & 1 & 1 & . & . & 1 & 1 & 1 & + \\
\hline Erica arborea & 2 & 1 & + & 1 & 1 & 1 & + & 1 & 1 & 2 & + & . & 1 & + & + & . & . & + & . & 1 & + \\
\hline
\end{tabular}




\begin{tabular}{|c|c|c|c|c|c|c|c|c|c|c|c|c|c|c|c|c|c|c|c|c|c|}
\hline Pteridium aquilinum & . & + & 1 & 2 & 2 & 2 & . & 1 & 2 & 3 & 2 & 3 & 2 & 1 & 2 & . & 1 & 3 & $\cdot$ & 2 & 2 \\
\hline Poa bulbosa & . & . & + & + & 1 & 1 & & 1 & 1 & 1 & 1 & 1 & 1 & 1 & 1 & . & 1 & 1 & 1 & 1 & 1 \\
\hline Agrostis castellana & 1 & 1 & 1 & 1 & 1 & 1 & $\cdot$ & 1 & 1 & 1 & 1 & 1 & 1 & 1 & 1 & $\cdot$ & + & + & 1 & 1 & + \\
\hline Cytisus scoparius & . & & + & + & 1 & 1 & . & 2 & 2 & 1 & 1 & . & 1 & 1 & 2 & . & . & + & . & 1 & 1 \\
\hline Genista florida & . & 1 & + & + & + & 1 & . & 1 & + & 1 & $\cdot$ & $\cdot$ & . & + & . & . & . & $\cdot$ & . & $\cdot$ & . \\
\hline Santolina oblongifolia & 1 & 1 & . & . & . & 1 & . & . & . & + & $\cdot$ & . & . & + & $\cdot$ & . & $\cdot$ & . & . & . & . \\
\hline Avenella iberica & 1 & 2 & 2 & 2 & 1 & 1 & . & 1 & 1 & 1 & 1 & 1 & 1 & 1 & 1 & 1 & 1 & 1 & 1 & 1 & + \\
\hline Pinus pinaster s.l. & . & $\cdot$ & 1 & . & 1 & 1 & . & . & 1 & 1 & & $\cdot$ & + & . & + & + & 1 & 1 & & 1 & . \\
\hline Rubus ulmifolius & . & $\cdot$ & + & + & . & + & . & + & + & . & $\cdot$ &. & . & . & 1 & 1 & 1 & + & 1 & 1 & . \\
\hline Crataegus monogyna & . & $\cdot$ & . & . & . & + & . & + & + & 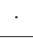 & $\cdot$ & + & . & . & + & + & + & . & 1 & . & · \\
\hline Rosa canina & . & $\cdot$ & . & . & . & $\cdot$ & . & $\cdot$ & . & $\cdot$ & $\cdot$ & $\cdot$ & . & . & $\cdot$ & + & 1 & $\cdot$ & 1 & 1 & . \\
\hline Quercus pyrenaica & . & $\cdot$ & . & + & 1 & 1 & . & . & . & . & $\cdot$ & . & . & . & + & . & + & $\cdot$ & . & $\cdot$ & . \\
\hline Daphne gnidium & · & $\cdot$ & . & . & . & 1 & . & 1 & . & + & $\cdot$ & . & . & · & + & . & $\cdot$ & $\cdot$ & . & $\cdot$ & · \\
\hline Thymus mastichina & . & $\cdot$ & + & + & . & . & $\cdot$ & $\cdot$ & . & + & $\cdot$ & & . & + & & . & $\cdot$ & 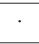 & $\cdot$ & . & . \\
\hline \multicolumn{22}{|l|}{ Others } \\
\hline Pilosella pseudopilosella & 1 & + & 1 & + & 1 & . & . & 1 & + & . & + & . & . & . & $\cdot$ & . & $\cdot$ & $\cdot$ & . & + & . \\
\hline Clinopodium vulgare & 1 & 1 & 1 & 1 & . & 1 & + & . & + & $\cdot$ & $\cdot$ & $\cdot$ & . & . & $\cdot$ & + & + & $\cdot$ & . & $\cdot$ & . \\
\hline Arrhenatherum elatius s.1. & . & $\cdot$ & . & . & + & 1 & . & 1 & 1 & 1 & 1 & + & 1 & + & 1 & . & $\cdot$ & + & 1 & 1 & + \\
\hline Cerastium ramosissimum & . & $\cdot$ & . & . & . & . & . & 1 & 1 & 1 & 1 & + & + & 1 & $\cdot$ & . & $\cdot$ & . & . & $\cdot$ & . \\
\hline $\begin{array}{l}\text { Rumex acetosella subsp. } \\
\text { angiocarpus }\end{array}$ & 1 & + & 1 & + & 1 & 1 & . & + & 1 & 1 & . & . & . & + & 1 & + & + & 1 & . & 1 & . \\
\hline Carduus carpetanus & + & + & + & . & 1 & 1 & + & 1 & 1 & 1 & $\cdot$ & + & . & . & 1 & + & + & 1 & . & 1 & . \\
\hline $\begin{array}{l}\text { Leucanthemopsis pallida } \\
\text { subsp. alpina }\end{array}$ & . & 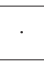 & . & . & . & . & . & . & + & 1 & 1 & . & . & . & . & . & . & . & . & . & . \\
\hline Armeria caespitosa & $\cdot$ & $\cdot$ & & & . & $\cdot$ & & & + & + & 1 & + & $\cdot$ & & & & $\cdot$ & $\cdot$ & $\cdot$ & & \\
\hline Pilosella castellana & + & 1 & 1 & + & 1 & . & $\cdot$ & 1 & $\cdot$ & + & 1 & 1 & 1 & 1 & $\cdot$ & $\cdot$ & $\cdot$ & & $\cdot$ & . & . \\
\hline Achillea millefolium & 1 & $\cdot$ & + & 1 & + & . & . & + & 1 & + & 1 & + & 1 & . & $\cdot$ & $\cdot$ & $\cdot$ & . & $\cdot$ & $\cdot$ & . \\
\hline Silene nutans & + & $\cdot$ & + & + & 1 & . & . & + & + & 1 & 1 & 1 & . & 1 & $\cdot$ & . & $\cdot$ & $\cdot$ & . & $\cdot$ & . \\
\hline Lotus corniculatus s.1. & 1 & 1 & 1 & . & . & . & . & . & + & + & $\cdot$ & · & . & . & $\cdot$ & . & $\cdot$ & $\cdot$ & . & $\cdot$ & . \\
\hline Anthoxanthum odoratum & . & $\cdot$ & $\cdot$ & . & . & . & . & $\cdot$ & + & 1 & 1 & 1 & 1 & 1 & 1 & . & $\cdot$ & + & $\cdot$ & $\cdot$ & + \\
\hline Arenaria montana & + & + & + & . & + & . & . & $\cdot$ & . & + & 1 & + & . & . & $\cdot$ & $\cdot$ & $\cdot$ & + & $\cdot$ & + & + \\
\hline Conopodium pyrenaeum & . & . & $\cdot$ & . & + & . & . & . & . & $\cdot$ & $\cdot$ & + & . & . & $\cdot$ & . & $\cdot$ & $\cdot$ & $\cdot$ & $\cdot$ & . \\
\hline Viola riviniana & + & 1 & + & + & + & . & . & . & . & + &. & + & . & . & $\cdot$ & . & $\cdot$ & . & . & . & . \\
\hline Ornithogalum concinnum & + & $\cdot$ & + & . & . & . & . & $\cdot$ & + & + & 1 & + & . & . & $\cdot$ & . & . & $\cdot$ & $\cdot$ & $\cdot$ & . \\
\hline Galium rotundifolium & + & 1 & + & + & . & . & . & + & + & + & $\cdot$ & $\cdot$ & . & . & 1 & . & . & + & 1 & 1 & . \\
\hline Merendera montana & 1 & 1 & . & . & . & . & . & . & . & . & . & . & . & . & . & . & . & $\cdot$ & . & . & . \\
\hline Corynephorus canescens & 1 & . & + & . & 1 & 1 & 2 & $\cdot$ & 1 & $\cdot$ & $\cdot$ & $\cdot$ & . & . & $\cdot$ & . & $\cdot$ & · & . & . & . \\
\hline Brachypodium sylvaticum & + & $\cdot$ & + & + & 1 & . & . & + & . & $\cdot$ & $\cdot$ & $\cdot$ & . & . & $\cdot$ & $\cdot$ & $\cdot$ & . & $\cdot$ & $\cdot$ & . \\
\hline Asplenium trichomanes & + & + & + & . & . & . & . & $\cdot$ & . & $\cdot$ & $\cdot$ & $\cdot$ & . & . & $\cdot$ & $\cdot$ & $\cdot$ & $\cdot$ & $\cdot$ & $\cdot$ & . \\
\hline Origanum vulgare & . & $\cdot$ & . & . & . & 1 & . & . & + & $\cdot$ & $\cdot$ & . & . & . & $\cdot$ & . & $\cdot$ & . & . & $\cdot$ & . \\
\hline Urtica dioica & . & $\cdot$ & . & . & . & $\cdot$ & . & $\cdot$ & + & . & $\cdot$ & + & . & . & $\cdot$ & + & + & $\cdot$ & . & $\cdot$ & . \\
\hline
\end{tabular}

Other species: shrubby, forest and perennials companions: Cistus laurifolius 1 and Cytisus multiflorus + in 34. Others: Carex elata subsp. reuteriana and Spergula morisonii + in 38; Ruscus aculeatus + in 54.

Localities: Ávila, Gredos range. 1: El Hornillo, Loma de la Majadilla, holotypus ass.; 2: El Hornillo, Silvona; 3: Guisando, La Bardera; 4: El Arenal, Arroyo de los Torneros; 5: El Arenal, Majomingo; 6-7: El Arenal, Arroyo de la Ortiga; 8: Navalacruz; 9: Casavieja, Garganta de la Chorrera; 10: Casavieja: Fuente de las Clavellinas; 11: Piedralaves, Garganta de la Graja. 12: Piedralaves, Los Hornillos; 13: La Adrada, Garganta del Charco de La Hoya; 14: La Adrada, Cueva de Majaltobar; 15: El Tiemblo, Las Juntas; 16-18: El Tiemblo, Cabeza de la Parra; 19-20: El Tiemblo, Fuente de las Víboras; 21: El Tiemblo, La Casquera.

Festuca elegans subsp. merinoi for clusters A2 and B2; and, finally, Pinus nigra subsp. salzmannii for clusters A1b and B2 (Table 3).

According to the rules proposed in the 'Material and Methods' section, Pinus nigra subsp. salzmannii was considered as differential of the cluster A1b (Echinosparto barnadesii-Pinetum ibericae pinetosum salzmannii), while Genista cinerascens and Festuca elegans subsp. merinoi were considered as diagnostic species of the cluster A2 (Festuco merinoi-Pinetum ibericae). Although in cases like that of Festuca elegans subsp. merinoi the differential role of the taxon is difficult to decide which vegetation unit is being differentiated ( $\Phi$ values: 0.251 cluster A2, 0.264 cluster B2), 
in cases like that of Pinus nigra subsp. salzmannii $(\Phi: 0.35)$ and Genista cinerascens $(\Phi: 0.368)$ is rather obvious their diagnostic character of cluster B2. These facts allow us to propose a new pinewood association for relevés of cluster B2: Genisto cinerascentis-Pinetum salzmannii ass. nova hoc loco (Table 6, holotypus, relevé 1).

Pinus nigra subsp. salzmannii dominated forests from calcareous and dolomitic substrates of the Iberian Peninsula have been usually classified within the orders of Quercetalia pubescentis and Prunetalia spinosae (QuercoFagetea class), Quercetalia ilicis (Quercetea ilicis class) and Ononido-Rosmarinetalia (Ononido-Rosmarinetea class) (Regato \& al., 1995; Rivas-Martínez \& al., 2001, 2002, 2011; Regato \& Del Río, 2009). Additionally, it was mentioned that Pinus nigra subsp. salzmannii forests from the oromediterranean and supramediterranean bioclimatic belts of southern Spain (Baza, Cazorla-Segura and Mágina ranges) and some from the southern Iberian System should be grouped under the Junipero sabinae-Pinetalia sylvestris order (PinoJuniperetea class) (Valle \& al., 1988; Regato \& al., 1995; Rivas-Martínez \& al., 2001, 2002). Given the floristic affinities between clusters A1-A2 and B2, which share some common diagnostic species such as Agrostis castellana, A. delicatula, Hypochoeris radicata, Jasione montana subsp. echinata, Genista cinerascens and Festuca elegans subsp. merinoi (Table 3), and accepting the concept of mutual ecological, biogeographical and structural characteristics, we believe that the Genisto cinerascentis-Pinetum salzmannii association should be put into Junipero sabinae-Pinetalia sylvestris order and Pino-Juniperetea class, which comprise mainly a group of western Mediterranean orophilous woody communities dominated by gymnosperms (RivasMartínez \& al., 2007, 2011). The structure of this vegetation community in the Gredos range is generally consisted by tree layer with more or less open canopies, thick shrub layer, and rather poor species assemblage (Table 6; Regato \& al., 1992). It also has relict character (Rubiales \& al., 2007, 2012; López-Sáez \& al., 2014). Spanish black pine has disappeared from many habitats of the Gredos range, mainly as a result of wildfires, excessive grazing and uncontrolled ploughing (Génova \& al., 1992, 2009; Cantero, 2004; Génova \& Moya, 2012), or interspecific competition, mostly with Pinus pinaster s.l. and Quercus pyrenaica (López-Sáez \& al., 2016a). These forests probably had a much wider distribution in the past, as shown by historical, dendrochronological and paleobotanical data (Génova \& al., 2008; Regato \& al., 1992, 1995; López-Sáez \& al., 2010, 2014).

The classification of the three sub-clusters of cluster B1 (Figure 2), with statistically determined diagnostic species for each subcluster, is presented in Table 3. Cluster B1a is well defined by Quercus pyrenaica, Galium rotundifolium and Rosa canina. This cluster features Pyrenean oak communities, which locally include relict Spanish black pines. Galán de Mera \& al. (1999) consider this fact as a thermal relict form of the Festuco merinoi -Quercetum pyrenaicae community between 1100 and $1350 \mathrm{~m}$ asl.

Cluster B1b shares some diagnostic species with Mediterranean distribution, such as Pinus pinaster s.1. and Daphne gnidium (Table 3), including $P$. pinaster s.l. dominated forests in the Gredos range that locally house Spanish black pines in the lower supramediterranean bioclimatic belt ( $\sim 900-1200 \mathrm{~m}$ asl). Probably, as palaeobotanical data have shown, the Spanish black pine had a wider distribution in the past occupying lower altitudinal levels at different times of the Holocene (Rubiales \& al., 2007, 2012; López-Sáez \& al., 2014). However, the gradual increase in temperature and competition with other species better adapted to fire regime and disturbances, such as Pinus pinaster s.1., reduced the presence of P. nigra subsp. salzmannii in lower areas of the Gredos range (López-Sáez \& al., 2010).

Cluster B1c represents relict populations of Spanish black pine that found refuge within alder (Alnus glutinosa) riparian forests $\sim 530$ $800 \mathrm{~m}$ asl. It is generally accepted that mountain pines (Pinus sylvestris s.l. and P. nigra s.1.) are Tertiary relicts with a continued presence in the Iberian Peninsula throughout the Quaternary (Alcalde \& al., 2006; Soto \& al., 2010). Although Pinus nigra subsp. salzmannii mainly grows today in the supramediterranean bioclimatic belt of the Iberian Peninsula, under humid or sub-humid conditions, it also grows at lower elevations in dry climate with temperate to extremely cold winter conditions and low annual precipitation (Regato \& al., 1995). In fact, one of the studied populations (sample 18) is located in the mesomediterranean bioclimatic belt at $530 \mathrm{~m}$ asl, being the popu- 
lation at lower altitudes in the whole entire Gredos mountains. Probably, these fragmented populations survived in situ during the last glacial maximum rather than having been recolonized in the post-glacial period, as shown by genetic studies (Afzal-Rafii \& Dodd, 2007; Desprat \& al., 2015).

\section{Acknowledgements}

This work was funded by the project HAR2013-43701-P (Spanish Economy and Competitiveness Ministry) "Dinámicas socioecológicas, resiliencia y vulnerabilidad en un paisaje de montaña: el Sistema Central (9000 cal. BC-1850 cal. AD) (DESIRE)"'. We are grateful to A. López Andreu (RIP), P. Sáez Navas and M. Tapia Ariza for their collaboration and assistance during the field work.

\section{Syntaxonomical scheme}

PINO-JUNIPERETEA Rivas-Martínez 1965

Pino sylvestris-Juniperetalia sabinae Rivas-Martínez 1965

Avenello ibericae-Pinion ibericae Rivas-Martínez \& J.A. Molina in Rivas-Martínez,

Fernández-González \& Loidi 1999

Echinosparto barnadesii-Pinetum ibericae Sánchez-Mata, Gavilán \& López-Sáez in López-

Sáez \& al. 2013

typicum Sánchez-Mata, Gavilán \& López-Sáez in López-Sáez \& al. 2013

pinetosum salzmannii Sánchez-Mata, Gavilán \& López-Sáez subass. nova

Festuco merinoi-Pinetum ibericae Sánchez-Mata, Gavilán \& López-Sáez in López-Sáez \& al. 2013

Genisto cinerascentis-Pinetum salzmannii Sánchez-Mata, Gavilán \& López-Sáez ass. nova

\section{Bibliography}

Abel-Schaad, D., Pulido, F.J., López-Sáez, J.A., Alba, F., Nieto, D., Franco, F., Pérez, S., Ruiz, M.B., Gil, M.J. \& Dorado, M. 2014. Persistence of tree relicts through the Holocene in the Spanish Central System. Lazaroa 35: 107-131.

Afzal-Rafii, Z. \& Dodd, R.S. 2007. Chloroplast DNA supports a hypothesis of glacial refugia over postglacial recolonization in disjunct populations of black pine (Pinus nigra) in western Europe. Mol. Ecol. 16: 723-736.

Alcalde, C., García-Amorena, I., García Álvarez, S., García Calvo, D., García García, R., Génova, M., Gil Borrell, P., Gómez Manzaneque, F., Maldonado, F.J., Morla, C., Del Nido, J., Postigo, J.M., Regato, P., Río, S., Roig, S., Rubiales, J.M. \& Sánchez Hernando, L.J. 2006-Contribución de la Paleofitogeografía a la interpretación del paisaje vegetal ibérico: estado de conocimientos y nuevas perspectivas de investigación. Inv. Agr. Sist. Rec. For. Fuera de Serie: 40-54.

Alía, R., Moro, J. \& Notivol, E. 2001. Genetic variability of Scots pine (Pinus sylvestris) provenances in Spain: growth traits and survival. Silva Fenn. 35: 27-38.

Arias, B., Climent, J.M. \& Díaz, P.M. 2011. Caracterización y diagnosis de Pinus nigra en Navalacruz (Ávila). Estrategias para la conservación de recursos genéticos y propuestas de gestión y mejora. Span. J. Rural Dev. 2: 151-160.

Aussenac, G. 1980. Comportement hydrique de rameaux excises de quelques espèces de sapins et de pins noirs en phase de desiccation. Ann. Sci. Forest. 37: 201-215.

Barbero, M., Loisel, R., Quézel, P., Richardson, D.M. \& Romane, F. 1998. Pines of the Mediterranean basin. In: Richardson, D.M. (Ed.). Ecology and biogeography of Pinus. Pp. 153-170. Cambridge Univ. Press. Cambridge.

Benito Garzón, M., Blazek, R., Neteler, M., Sánchez de Dios, R., Sainz Ollero, H. \& Furlanello, C. 2006. Predicting habitat suitability with machine learning models: The potential area of Pinus sylvestris L. in the Iberian Peninsula. Ecol. Model. 197: 383-393.

Benito Garzón, M., Sánchez de Dios, R. \& Sainz Ollero, H. 2008a. Effects of climate change on the distribution of Iberian tree species. Appl. Veg. Sci. 11: 169-178.

Benito Garzón, M., Sánchez de Dios, R. \& Sainz Ollero, H. 2008b. The evolution of the Pinus sylvestris L. area in the Iberian Peninsul from the last glacial máximum to 2100 under climate change. Holocene 18: 705-714. 
Blanco Castro, E., Casado González, M.A., Costa Tenorio, M., Escribano, R., García Antón, M., Génova Fuster, M., Gómez Manzaneque, A., Gómez Manzaneque, F., Moreno Saiz, J.C., Morla Juaristi, C., Regato Pajares, P. \& Sainz Ollero, H. 1997. Los bosques ibéricos. Una interpretación geobotánica. Planeta. Barcelona.

Boratynski, A. 1991. Range of natural distribution. In: Giertych, M. \& Mátyás, C. (Eds.). Genetics of Scots Pine. Pp. 19-30. Akadémiai Kiadó. Budapest.

Braun-Blanquet, J. 1979. Fitosociología. Bases para el estudio de las comunidades vegetales. H. Blume. Barcelona.

Bruelheide, H. 2000. A new measure of fidelity and its application to defining species groups. J. Veg. Sci. 11: $167-178$.

Cantero, A. 2004. Una joya desprotegida: los pinos relícticos de la vertiente sur de la Sierra de Gredos (Ávila). Montes 76: 35-40.

Castroviejo, S. \& al. (Coord.). 1986-2015. Flora iberica. Plantas vasculares de la Península Ibérica e Islas Baleares. Vols. I-XXI. R. Jard. Bot. CSIC. Madrid.

Catalán, G., Gil, P., Galera, R., Martín, S., Agúndez, D. \& Alía, R. 1991. Las regiones de procedencia de Pinus sylvestris L. y Pinus nigra Arn. subsp. salzmannii (Dunal) Franco en España. ICONA. Madrid.

Chytrý, M., Tichý, L., Holt, J. \& Botta-Dukát, Z. 2002. Determination of diagnostic species with statistical fidelity measures. J. Veg. Sci. 13: 79-90.

Costa, M., García Antón, M., Morla, C. \& Sainz Ollero, H. 1990. La evolución de los bosques de la Península Ibérica: una interpretación basada en datos paleobiogeográficos. Ecología 1: 31-58.

De la Cruz, M. \& Peinado, M. 1996. El paisaje vegetal de la Cuenca del río Henares. I. Comunidades arbóreas y arbustivas. Wad-al-Hayara 23: 335-396.

De la Fuente, V. \& Ortúñez, E. 2001. Festuca sect. Eskia (Poaceae) in the Iberian Peninsula. Folia Geobot. 36: 385-421.

Desprat, S., Díaz Fernández, P., Coulon, T., Ezzat, L., Pessarossi-Langlois, J., Gil, L., Morales-Molino, C. \& Sánchez Goñi, M.F. 2015. Pinus nigra (Spanish black pine) as the dominant species of the last glacial pinewoods in south-western to central Iberia: a morphological study of modern and fossil pollen. J. Biogeogr. 42: 1998-2009.

Elena Rosselló, R. \& Sánchez Palomares, O. 1991. Estudio ecológico de los pinares españoles de Pinus nigra Arn.: síntesis ecológica. Monogr. INIA 81. Madrid.

Elena Rosselló, R., Sánchez Palomares, O. \& Carretero, P. 1985. Estudio fisiográfico y climático de los pinares autóctonos españoles de Pinus nigra Arn. Com. INIA Ser. Rec. Nat. 36. Madrid.

Escudero, A., Gavilán, R. \& Rubio, A. 1994. Una breve revisión de técnicas de análisis multivariantes aplicables en Fitosociología. Bot. Complutensis 19: 9-38.

Galán de Mera, A., Hagen, M.A. \& Vicente Orellana, J.A. 1999. Plant communities with Pinus sylvestris L. and P. nigra Arnold subsp. salzmannii (Dunal) Franco of the Spanish Sistema Central: a phytosociological approximation. Bot. Helv. 109: 21-54.

Gamisans, J. \& Gruber, M. 1988. Els boscos de Pinassa (Pinus nigra subsp. salzmannii) als Pirineus catalans i est-aragonesos: estudi fitosociologic. Monogr. Inst. Piren. Jaca 4: 543-552.

Gamisans, J., Gruber, M. \& Regato, P. 1991. Les fôrets de Pinus nigra subsp. salzmannii des Pyrénées aragonaises. Lazaroa 12: 147-151.

Gandía, R., Iglesias, A. \& Martín, J.M. 2005. Los recursos genéticos de Pinus nigra Arnold subsp. salzmannii (Dunal) Franco en la Sierra de Gredos, diario de su conservación. In: Grande, M. \& García, A. (Eds.). Los pinares de Pinus nigra Arn. en España: Ecología, uso y gestión. Pp. 643-664. Fundación Conde del Valle de Salazar. Madrid.

García López, J.M., Gonzalo Jiménez, J. 6 Allué, C. 2005. Fitoclimatología de Pinus nigra Arn. subsp. salzmannii (Dunal) Franco en España. Áreas potenciales de ensayo y adecuaciones fitoclimáticas. In: Grande, M. \& García, A. (Eds.). Los pinares de Pinus nigra Arn. en España: Ecología, uso y gestión. Pp. 85-108. Fundación Conde del Valle de Salazar. Madrid.

Gavilán, R. 2005. The use of climatic parameters and indices in vegetation distribution. A case study in the Spanish Sistema Central. Int. J. Biometeorol. 50: 111-120.

Gavilán, R., Fernández-González, F. \& Blasi, C. 1998. Climatic classification and ordination of the Spanish Sistema Central: relationships with potential vegetation. Plant Ecol. 139: 1-11.

Gea-Izquierdo, G., Montes, F., Gavilán, R.G., Cañellas, I. \& Rubio, A. 2015. Is this the end? Dynamics of a relict stand from pervasively deforested ancient Iberian pine forests. Eur. J. For. Res. 134: 525-536.

Génova, M., García Amorena, I., Gómez Manzaneque, F., Rubiales, J.M. \& Morla, C. 2008. Cronologías milenarias de Pinus en la Sierra de Gredos. In: Redondo, M.M., Palacios, M.T., López, F.J., Santamaría, T. \& Sánchez Mata, D. (Eds.). Avances en Biogeografía. Pp. 143-145. Min. Educ. Ci. Madrid. 
Génova, M., Gómez Manzaneque, F. \& Morla Juaristi, C. 2009. Los bosques de Gredos a través del tiempo. Junta de Castilla y León. Valladolid.

Génova, M.M., Gómez-Manzaneque, F. \& Regato, P. 1992. Sobre los pinares relictos de la Sierra de Gredos (Ávila). In: Actes del Simposi Internacional de Botànica "Pius Font i Quer"- Vol. II Fanerogàmia. Pp. 439-442. Inst. Est. Ilerd. Lleida.

Génova, M. \& Moya, P. 2012. Dendroecological analysis of relict pine forests in the centre of the Iberian Peninsula. Biodivers. Conserv. 21: 2949-2965.

Gil, L., Galera, R.M., Martín, S., Agúndez, D. \& Alía, R. 1991. Las regiones de procedencia de Pinus sylvestris L. y Pinus nigra Arn. subsp. salzmannii (Dunal) Franco en España. ICONA. Madrid.

Gómez Manzaneque, F. 1988. Algunos taxones interesantes del suroeste madrileño. Stud. Bot. 7: 257-261.

González Molina, J.M. 2006. Manual de gestión de los hábitats de pino silvestre en Castilla y León. Cons. Med. Amb. Junta Castilla y León. Valladolid.

Lepš, J. \& Šmilauer, P. 2003. Multivariate analysis of Ecological Data using CANOCO $^{\mathrm{TM}}$. Cambridge Univ. Press. Cambridge.

Loidi, J., Biurrun, I. \& Herrera, M. 1997a. La vegetación del centro-septentrional de España. Itinera Geobot. 9: 161-168.

Loidi, J., Díaz, T. \& Herrera, M. 1997b. El paisaje vegetal del norte-centro de España. Guía de la excursión. Itinera Geobot. 9: 5-160.

López-Leiva, C., Espinosa, J. \& Bengoa, J. 2009. Mapa de vegetación de Castilla y León. Síntesis 1:400.000. Junta de Castilla y León Valladolid.

López-Merino, L., López-Sáez, J.A., Alba-Sánchez, F., Pérez-Díaz, S. \& Carrión, J.S. 2009. 2000 years of pastoralism and fire shaping high-altitude vegetation of Sierra de Gredos in central Spain. Rev. Palaeobot. Palynol. 158: 42-51.

López-Sáez, J.A., Abel, D., Pérez, S., Blanco, A., Alba, F., Dorado, M., Ruiz, B., Gil, M.J., Gómez, C. \& Franco, F. 2014. Vegetation history, climate and human impact in the Spanish Central System over the last 9,000 years. Quat. Int. 353: 98-122.

López-Sáez, J.A., Abel, D., Robles, S., Pérez, S., Alba, F. \& Nieto, D. 2016a. Landscape dynamics and human impact on high-mountain woodlands in the western Spanish Central System during the last three millennia. J. Archaeol. Sci.: Rep. 9: 203-218.

López-Sáez, J.A., Alba, F., Robles, S., Pérez, S., Abel, D., Sabariego, S. \& Glais, A. 2016b. Exploring seven hundred years of transhumance, climate dynamic, fire and human activity through a historical mountain pass in central Spain. J. Mt. Sci. 13: 1139-1153.

López-Sáez, J.A. \& López-García, P. 1994. Contribution of the palaeoecological knowledge of Quaternary in the Tietar Valley (Sierra de Gredos, Ávila, Spain). Rev. Esp. Micropal. 26: 61-66.

López-Sáez, J.A., López-Merino, L., Alba-Sánchez, F. \& Pérez-Díaz, S. 2009. Contribución paleoambiental al estudio de la trashumancia en el sector abulense de la Sierra de Gredos. Hispania 69: 9-38.

López-Sáez, J.A., López-Merino, L., Alba-Sánchez, F., Pérez-Díaz, S., Abel-Schaad, D. \& Carrión, J.S. 2010. Late Holocene ecological history of Pinus pinaster forests in the Sierra de Gredos of central Spain. Plant Ecol. 206: 195-209.

López-Sáez, J.A., Sánchez-Mata, D., Alba, F., Abel, D., Gavilán, R.G. \& Pérez Díaz, S. 2013. Discrimination of Scots pine forests in the Iberian Central System (Pinus sylvestris var. iberica) by means of pollen analysis. Phytosociological considerations. Lazaroa 34: 191-208.

Mancebo, J.M., Molina, J.R. \& Camino, F. 1993. Pinus sylvestris L. en la vertiente septentrional de la sierra de Gredos (Ávila). Ecología 7: 233-245.

Martínez-García, F. \& Montero, G. 2000. Typology of Pinus sylvestris L. forests in Spain. Inv. Agrar. Sist. Rec. For. Fuera Ser. 1: 41-65.

Molina Moreno, J.R. 1992. Flora y vegetación del Valle de Iruelas (Ávila). Cuadernos Abulenses 18: 11-149.

Regato, P. \& Del Río, M. 2009. 9530. Pinares (sud-) mediterráneos de Pinus nigra endémicos. In: VV.AA. (Eds.). Bases ecológicas preliminares para la conservación de los tipos de hábitat de interés comunitario en España. Pp. 1-95. Min. Med. Amb. Med. Rur. Mar. Madrid.

Regato, P. \& Elena Rosselló, R. 1995. Natural black pine (Pinus nigra subsp. salzmannii) forests of the Iberian eastern mountains: development of the phytoecological basis for their site evaluation. Ann. Sci. For. 52: 589-606.

Regato, P., Elena Rosselló, R. \& Sánchez Palomares, O. 1991. Estudio autoecológico comparativo de Pinus nigra Arn. subsp. salzmannii de la Península Ibérica y otras subespecies de la región circunmediterránea. Inv. Agr. Sist. Rec. For. 0: 49-59.

Regato, P. \& Escudero, A. 1989. Caracterización fitoecológica de las comunidades de Pinus nigra subsp. salzmannii en los afloramientos rocosos del Sistema Ibérico meridional. Bot. Complut. 15: 149-161.

Regato, P., Génova, M. \& Gómez Manzaneque, F. 1992. Las representaciones relictas de Pinus nigra Arnold en el Sistema Central español. Bol. R. Soc. Esp. Hist. Nat. (Biol.) 88: 63-71. 
Regato, P., Gamisans, J. \& Gruber, M. 1995. A syntaxonomical study of Pinus nigra subsp. salzmannii forests in the Iberian Peninsula. Phytocoenologia 25: 561-578.

Rivas-Martínez, S. 1987. Memoria del MAPA de Series de Vegetación de España, escala 1:400.000. MAPA \& ICONA. Madrid.

Rivas-Martínez, S. \& al. 2007. Mapa de series, geoseries y geopermaseries de vegetación de España [Memoria del mapa de la vegetación potencial de España], parte I. Itinera Geobot. 17: 5-436.

Rivas-Martínez, S. \& al. 2011. Mapa de series, geoseries y geopermaseries de vegetación de España [Memoria del mapa de la vegetación potencial de España], parte II. Itinera Geobot. 18: 5-800.

Rivas-Martínez, S., Díaz, T.E., Fernández-González, F., Izco, J., Loidi, J., Lousã, M. \& Penas, A. 2002. Vascular plant communities of Spain and Portugal. Addenda to the Syntaxonomical checklist of 2001. Itinera Geobot. 15: 5-922.

Rivas-Martínez, S., Fernández-González, F., Loidi, J., Lousã, M. \& Penas, A. 2001. Syntaxonomical checklist of vascular plant communities of Spain and Portugal to association level. Itinera Geobot. 14: 5-341.

Romera García, E., Fernández Cancio, A., Grau Corbí, J.M. \& Bautista Carrascosa, R. 2001. Límites y clasificación fitoclimática de Pinus nigra Arnold. Cuad. Soc. Esp. Cien. For. 12: 99-108.

Rubiales, J.M., García-Amorena, I., Génova, M., Gómez-Manzaneque, F. \& Morla, C. 2007. The Holocene history of highland pine forests in a submediterranean mountain: the case of Gredos mountain Range (Iberian Central Range). Quat. Sci. Rev. 26: 1759-1770.

Rubiales, J.M., Morales-Molino, C., García, S. \& García-Antón, M. 2012. Negative responses of highland pines to anthropogenic activities in inland Spain: a palaeoecological perspective. Veg. Hist. Archaeobot. 21: 397-412.

Ruby, J.L. \& Wright, J.W. 1976. A revised classification of geographic varieties in Scots pine. Silvae Genet. 25: 169-175.

Sánchez-Mata, D. 1989. Flora y vegetación del Macizo Oriental de la Sierra de Gredos (Ávila). Institución Gran Duque de Alba. Ávila.

Sánchez-Mata, D. 1999. Bioclimatología: Una ciencia avanzada para la caracterización del medio natural. Discursos Entrada 1998. Pp. 95-112. Institución Gran Duque de Alba. Ávila.

Sánchez-Mata, D. 2015. Hábitats y vegetación natural en la alta montaña del Parque Regional de la Sierra de Gredos (Castilla y León, Ávila). Discurso Ingreso Acad. Farmacia Castilla y León. CERSA. Salamanca.

Soto, A., Robledo Arnuncio, J.J., González Martínez, S.C., Smouse, P.E. \& Alía, R. 2010. Climatic niche and neutral genetic diversity of the six Iberian pin especies: a retrospective and prospective view. Mol. Ecol. 19: 1396-1409.

ter Braak, C.J.F. \& Šmilauer, P. 2002. CANOCO Reference Manual and Canodraw for Windows User's Guide. Software for Canonical Community Ordination. Biometris. Wageningen.

Tichý, L. 2002. JUICE, software for vegetation classification. J. Veg. Sci. 13: 451-453.

Tichý, L. \& Chytrý, M. 2006. Statistical determination of diagnostic species for site groups of unequal size. J. Veg. Sci. 17: 809-818.

Tichý, L., Chytrý, M. \& Šmarda, P. 2011. Evaluating the stability of the classification of community data. Ecography 34: 807-813.

Tutin, T.G., Heywood, V.H., Burges, N.A., Moore, D.M., Valentine, D.H., Walters, S.M. \& Webb, D.A. 1964-1980. Flora Europaea, 5 vols.. Cambridge Univ. Press. Cambridge.

Valle, F., Mota, J.F. \& Gómez Mercado, F. 1988. Datos sobre la vegetación orófila de Andalucía oriental (España). Doc. Phytosociol. 11: 459-464.

Vigo, J. 1979. Les forêts de conifères des Pyrénées catalanes: essai de revisión phytocenologique. Doc. Phytosociol. 4: 929-941.

Ward, J.H. 1963. Hierarchical grouping to optimize an objective function. J. Am. Stat. Assoc. 58: 236-244.

Wildi, O. 1989. A new numerical solution to traditional phytosociological tabular classification. Vegetatio 81: 95-106.

\section{Floristic Appendix}

Avenella iberica (Rivas Mart., Izco \& Costa) Rivas Mart.

Cytisus striatus (Hill) Rothm. subsp. eriocarpus (Boiss. \& Reut.) Rivas Mart.

Festuca elegans Boiss. subsp. merinoi (Pau) Fuente \& Ortúñez

Festuca gredensis Fuente \& Ortúñez

Jasione montana L. subsp. echinata (Boiss. \& Reut.) Rivas Mart.

Festuca paniculata (L.) Schinz \& Thell. subsp. multispiculata Rivas Ponce \& Cebolla

Leucanthemopsis pallida (Mill.) Heywood subsp. alpina (Boiss. \& Reut.) Rivas Mart., Fern. Gonz. \&

Sánchez-Mata

Pinus sylvestris L. var. iberica Svoboda 